\title{
SÍNTESE E CARACTERIZAÇÃO DE COPOLÍMEROS DE CADEIA LATERAL DERIVADOS DE ACRILATOS DE 4,5-DI-HIDROISOXAZOL E DO (-)-MENTOL
}

\author{
Joel A. Passo e Aloir A. Merlo*\# \\ Instituto de Química, Universidade Federal do Rio Grande do Sul, Av. Bento Gonçalves, 9500, 91501-970 Porto Alegre - RS, Brasil \\ Juliana Eccher e Ivan H. Bechtold \\ Departamento de Física, Universidade Federal de Santa Catarina, Campus Universitário Trindade, 88040-900 Florianópolis - SC, \\ Brasil \\ Stephen M. Kelly \\ Department of Chemistry, University of Hull, U67RX, Hull, UK
}

Recebido em 28/10/11; aceito em 30/3/12; publicado na web em 10/7/12

\begin{abstract}
SYNTHESIS AND CHARACTERIZATION OF COPOLYMERS 4,5-DIHYDROISOXAZOLE AND (-)-MENTHYL ACRYLATES. Five monomers 5-[4-(5-cyano-4,5-dihydroisoxazol-3-yl)phenoxy]undecyl acrylate (7a); $n$-alkyl 3-\{4-[5-(acryloyloxyundecyl) oxyphenyl] \}-4,5-dihydroisoxazole-5-carboxylate (7b,c for $n$-butyl and $n$-hexyl, respectively); 3-\{4-[5-(acryloyloxyundecyl) oxyphenyl] \}-4,5-dihydroisoxazole-5-carboxylic acid (7d) and $(1 R, 2 S, 5 R)$-2-isopropyl-5-methylcyclohexyl acrylate (9) and the corresponding copolymers 10a-d, 11 and homopolymers 12 from 7a and $\mathbf{1 3}$ from $\mathbf{9}$ were designed and synthesized. Except for acrylate $\mathbf{9}$ which is derived from (-)-menthol, all of the monomers belong to the series containing the isoxazoline ring linked to the acrylate unit by a flexible spacer chain of eleven methylene units. They presented low glass temperature and despite birefringence behavior, these copolymers showed no mesomorphic properties.
\end{abstract}

Keywords: 3,5-disubstituted isoxazolines; copolymers; menthyl acrylate.

\section{INTRODUÇÃO}

Copolímeros são macromoléculas compostas por duas ou mais unidades estruturais diferentes, que podem se distribuir estatisticamente entre si ou de forma a gerar segmentos ou blocos. ${ }^{1}$ Podem ser classificados quanto à forma, tendo como base a organização das unidades ao longo da cadeia polimérica, em alternados, periódicos, estatísticos, aleatórios ou em blocos. Também são classificados em lineares ou ramificados, dependendo do arranjo da cadeia polimérica. ${ }^{2}$

A reação de copolimerização é uma técnica importante que possibilita modular o polímero, através de modificações sistemáticas, agregando propriedades específicas, tornando-o comercial e tecnologicamente mais interessante e vantajoso. ${ }^{3}$ Como exemplo, pode-se citar a quitina ${ }^{4}$ e seu derivado, a quitosana. Esses são poliaminossacarídeos que têm uma vasta aplicação no campo biomédico. No entanto, esses polímeros naturais mostram algumas desvantagens no uso comercial, por serem insolúveis nos principais solventes utilizados cotidianamente. A fim de superar esse problema pode-se copolimerizar o poliaminossacarídeo com monômeros vinílicos produzindo copolímeros multifuncionais, alterando desta forma as propriedades físico-químicas do polímero natural. Os copolímeros são utilizados de forma bastante diversificada em pervaporação organofílica, ${ }^{5}$ formulações para recuperação de óleos terciários, projetos hidráulicos, ${ }^{6}$ microgéis inteligentes com aplicação em diversas áreas, tais como transportadores de medicamentos, sensores, separadores químicos e imobilizadores de enzimas. ${ }^{7}$

$\mathrm{Na}$ área de cristais líquidos, a copolimerização é uma ferramenta importante para preparação de novos materiais que apresentam as propriedades mesogênicas combinadas com as dos materiais poliméricos relacionadas com elasticidade, processabilidade, formação de filmes,

*e-mail: aloir@iq.ufrgs.br

\#Endereço atual: Departamento de Química, Universidade de Hull, Inglaterra, HU67RX confinamento, entre outras. ${ }^{8}$ Neste contexto, estamos interessados na síntese e caracterização de novos materiais líquido-cristalinos contendo anéis heterocíclicos, em particular, os anéis "primos" isoxazolina e isoxazol ${ }^{9}$ apresentando diferentes substituintes nas posições 3 e 5, respectivamente. Essa característica estrutural confere à molécula uma forma geométrica dobrada em $\mathrm{V}$, com a proporção comprimento:largura $(l / d)$ entre o eixo molecular longitudinal $(l)$ e o o eixo transversal $(d)$ apropriada para mostrar comportamento anisotrópico. Esse fator não é o único que se deve considerar na área de cristais líquidos, mas é fundamental que seja obedecida a relação $l>d$. Em trabalhos anteriores, verificamos que mesofases enantiotrópicas com ampla faixa de temperatura surgiram apenas no polímero de cadeia lateral final a partir de monômeros acrilatos 3,5-diarilisoxazolínicos. ${ }^{9}$ Para os monômeros precursores, apenas em alguns casos se observaram mesofases monotrópicas. Comportamento similar também foi observado num conjunto de três séries homólogas não poliméricas publicadas recentemente. ${ }^{10} \mathrm{O}$ resultado encontrado está de acordo com os trabalhos pioneiros desenvolvidos por Finkelmann e colaboradores na área de cristais líquidos poliméricos de cadeia lateral (CLPCL). ${ }^{11}$ Compostos mesogênicos poliméricos e não poliméricos também têm sido estudados em decorrência das potencialidades aplicativas em dispositivos que refletem seletivamente a luz, termocrômicos etc.. ${ }^{12,13}$

Neste trabalho, descreve-se a síntese e caracterização de novos materiais poliméricos de cadeia lateral obtidos da combinação de monômeros acrilatos derivados de isoxazolinas com um segundo monômero acrilato quiral derivado do mentol. No entanto, os monômeros isoxazolínicos sintetizados neste trabalho apresentam forma geométrica reduzida, ou seja, a proporção comprimento:largura $(l / d)$ é menor quando são comparados a monômeros sintetizados em trabalhos anteriores. ${ }^{9}$ Para este trabalho, empregou-se o substituinte na posição 5 do anel isoxazolínico e um grupo carboxílico, carboxilato e ciano, sendo não aromático e de tamanho reduzido. O propósito é testar a capacidade de indução do comportamento mesomórfico durante o evento da polimerização, a partir dos monômeros isoxazolínicos 
com uma dimensão molecular reduzida $(l / d)$ e na presença de um segundo monômero acrilato quiral. A redução de $l$ é devida à ausência do substituinte arila na posição 5 do anel isoxazolínico. Se afirmativo, verificar se as possíveis mesofases formadas apresentam alguma torção helicoidal, devido à presença de segmentos quirais dispersos lateralmente na cadeia polimérica, bem como avaliar a natureza das mesofases formadas. ${ }^{14} \mathrm{~A}$ utilização de monômeros quirais derivados do colesterol seria uma alternativa interessante para avaliar o efeito da quiralidade..$^{15}$ No entanto, os ésteres colestéricos são naturalmente cristais líquidos, o que mascararia a capacidade indutora de formação de mesofases do anel isoxazolínico.

A Figura 1 descreve genericamente os copolímeros preparados. Os grupos laterais dispersos ao longo da cadeia polimérica representam os grupos derivados do anel isoxazolínico separados por um espaçador flexível e da mentila ligada diretamente ao grupo acrilato. A razão geométrica $l / d$ responsável pela anisometria de forma também é mostrada.

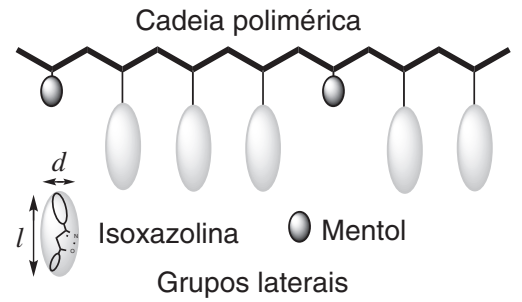

Figura 1. Estrutura genérica do copolímero acrilato contendo cadeias laterais derivadas de isoxazolina e mentol. A anisotropia geométrica indicada pela razão l/d é mostrada

\section{RESULTADOS E DISCUSSÃO}

Os Esquemas de síntese 1 e 2 descrevem as estratégias de preparação dos monômeros 7a-d e 9, dos copolímeros 10a-d e $\mathbf{1 1}$ e dos homopolímeros 12 e $\mathbf{1 3}$.

A obtenção dos monômeros 3-arilisoxazolina 7a-d é descrita no Esquema 1. A reação chave é a cicloadição [3+2] 1,3-dipolar de óxidos de nitrilas e dipolarófilos com grupos aceptores de elétrons $\mathrm{Z}$. A síntese foi iniciada com a reação de alquilação do aldeído $\mathbf{1}$ com o reagente 11-bromo-1-undecanol, com $79 \%$ de rendimento de $\mathbf{2}$. $\mathrm{Na}$ sequência foi sintetizada a oxima $\mathbf{3}$, a partir da reação do aldeído $\mathbf{2}$ com cloridrato de hidroxilamina $\left(\mathrm{AcONa} ; \mathrm{EtOH} / \mathrm{H}_{2} \mathrm{O}\right) \operatorname{com} 72 \%$ de

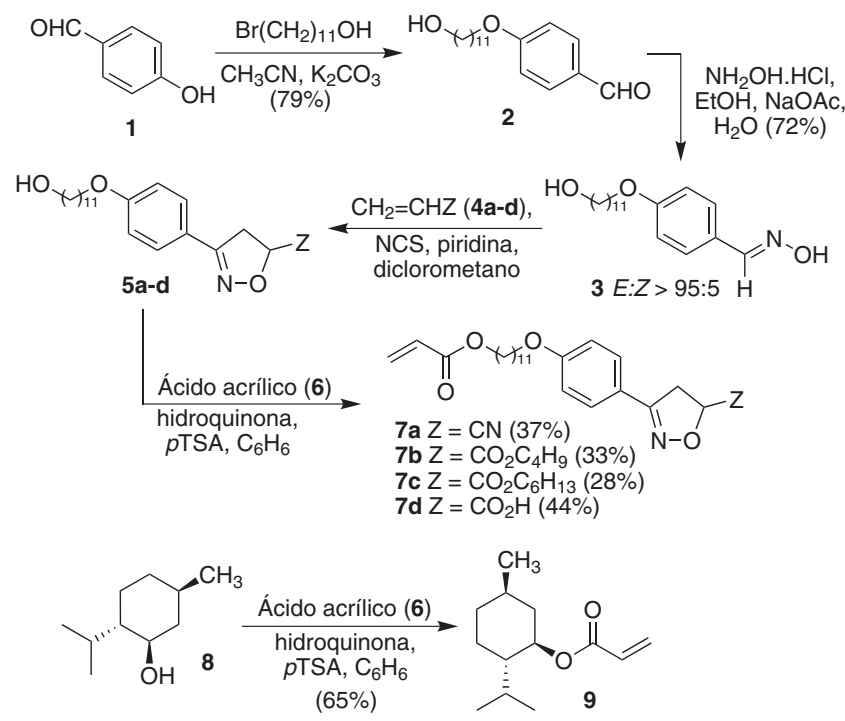

Esquema 1. Síntese dos monômeros 7a-d e 9 rendimento. As oximas foram obtidas na forma de mistura de isômeros $E: Z$. A proporção dos isômeros $E: Z$ foi de $>95: 5$ a favor do isômero $E$, de acordo com a atribuição feita a partir do sinal observado no espectro de $\mathrm{RMN}{ }^{1} \mathrm{H}$ do hidrogênio imínico $\mathrm{H}-\mathrm{C}=\mathrm{NOH}$ relacionado com os isômeros $E$ - e $Z$-, respectivamente. ${ }^{16}$

A síntese das isoxazolinas 5a-d foi feita a partir da cicloadição [3+2] 1,3-dipolar ${ }^{17}$ do óxido de nitrila gerado in situ da respectiva oxima 3 e os dipolarófilos 4a-d, com rendimentos na faixa de 40-70\%. Finalmente, através da reação de esterificação dos intermediários 5a-d com excesso de ácido acrílico (6), na presença de ácido $p$-tolueno sulfônico e de hidroquinona, produziram-se os monômeros 7a-d com rendimentos de médios para baixos.

A análise dos espectros de $\mathrm{RMN}{ }^{1} \mathrm{H}$ e ${ }^{13} \mathrm{C}$ dos intermediários 7a-d confirmou a formação do regioisômero 3,5 em detrimento do regioisômero 3,4 na reação de cicloadição. No espectro de hidrogênio observou-se o sinal do hidrogênio ligado ao $\mathrm{C}_{3}$ em 5,1 ppm e os sinais dos hidrogênios ligados aos carbonos 4 e 5 em 3,6 ppm, confirmando a regioquímica do anel isoxazolínico 3,5-dissubstituído. No espectro de $\mathrm{RMN}{ }^{13} \mathrm{C}$ da isoxazolina $\mathbf{5 b}$ foram observados os sinais referentes ao anel isoxazolínico 3,5-substituído em 39,0 ppm relativo ao $\mathrm{C}_{4}$ do anel, 77,7 ppm relativo ao $\mathrm{C}_{5}$ e em $155,5 \mathrm{ppm}$ relativo ao $\mathrm{C}_{3} \cdot{ }^{18}$

Análises dos espectros de RMN ${ }^{1} \mathrm{H},{ }^{13} \mathrm{C}$ e IV confirmaram a inserção do grupo polimerizável acrilato na estrutura final. Na Figura 2 podem-se observar, na parte em destaque do espectro expandido de $\mathrm{RMN}{ }^{1} \mathrm{H}$ do composto $\mathbf{7 b}$, os sinais do grupo acrilato na região espectral entre 5,5 a 6,5 ppm. A multiplicidade observada é atribuída aos hidrogênios olefínicos $\mathrm{H}_{1}, \mathrm{H}_{2}$ e $\mathrm{H}_{3}$ pertencentes ao sistema AMX, localizados em 5,8; 6,1 e 6,4 ppm com ${ }^{3} J_{\text {trans }},{ }^{3} J_{\text {cis }} \mathrm{e}^{2} J_{\text {gem }}$ da ordem de 17,$3 ; 10,4$ e 1,6 Hz, respectivamente. No espectro de carbono C-13, podem-se observar os sinais dos carbonos da ligação dupla do grupo acrilato em 128,5 e 130,4 ppm. Também se observa o sinal do carbono carbonílico em 166,5 ppm, junto com os demais sinais referentes ao intermediário $\mathbf{7 b}$, confirmando a inserção do grupo acrilato.

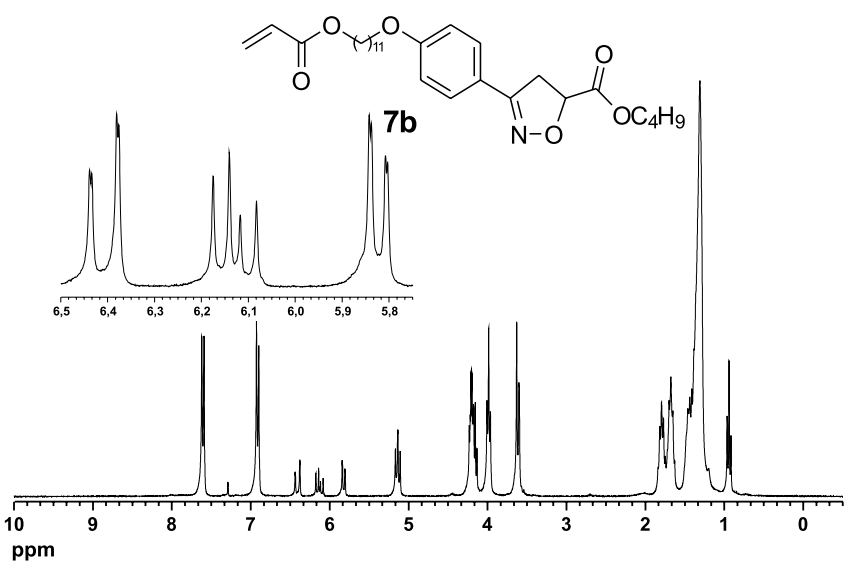

Figura 2. Espectro $R M N$ de ${ }^{1} \mathrm{H}$ do monômero $7 \boldsymbol{b}$

O monômero quiral 9 foi produzido com rendimento de $65 \%$ a partir do (-)-mentol (8) seguindo o mesmo protocolo reacional dos monômeros descrito no Esquema 1.

Os copolímeros 10a-d e $\mathbf{1 1}$ (Esquema 2) foram produzidos via polimerização radicalar com AIBN em tolueno a $65^{\circ} \mathrm{C}$ nas proporções em mol de 70:30 e 90:10, respectivamente, de acordo com a Tabela 1. A purificação dos copolímeros foi feita pela extração contínua do monômero residual que não reagiu, através de um extrator de Soxhlet. De tempos em tempos, uma alíquota era removida e devidamente analisada via ressonância de $\mathrm{RMN}{ }^{1} \mathrm{H}$, acompanhando o desaparecimento dos sinais dos hidrogênios olefínicos do grupo acrilato. As proporções entre os monômeros nos copolímeros foram confirmadas 
por análise de $\mathrm{RMN}{ }^{1} \mathrm{H}$, comparando as áreas relativas de picos na faixa de 5.4 e $4.6 \mathrm{ppm}$ da parte isoxazolínica e da parte do grupo mentila, respectivamente.

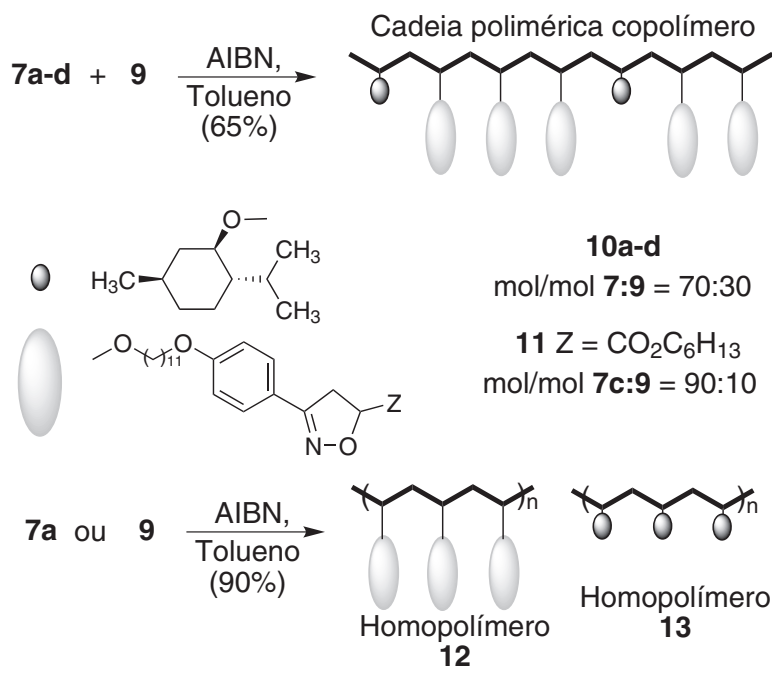

Esquema 2. Síntese dos copolímeros 10a-d e 11 e dos homopolímeros 12 e 13

Tabela 1. Dados dos copolímeros 10a-d e 11

\begin{tabular}{cccc}
\hline Copolímeros $^{\mathrm{a}}$ & $-\mathrm{Z}$ & 7a-d:9 & Rend (\%) \\
\hline $\mathbf{1 0 a}$ & $-\mathrm{CN}$ & $71: 29$ & 40 \\
$\mathbf{1 0 b}$ & $-\mathrm{CO}_{2} \mathrm{C}_{4} \mathrm{H}_{9}$ & $51: 49$ & 54 \\
$\mathbf{1 0 c}$ & $-\mathrm{CO}_{2} \mathrm{C}_{6} \mathrm{H}_{13}$ & $71: 29$ & 57 \\
$\mathbf{1 0 d}$ & $-\mathrm{CO}_{2} \mathrm{H}$ & $85: 15$ & 30 \\
$\mathbf{1 1}$ & $-\mathrm{CO}_{2} \mathrm{C}_{6} \mathrm{H}_{13}$ & $86: 14$ & 64 \\
\hline
\end{tabular}

${ }^{a}$ Proporção em mol:mol obtido via espectros de $\mathrm{RMN}{ }^{1} \mathrm{H}$.

Para efeito comparativo foram preparados os homopolímeros poliacrilato 12, contendo o grupo terminal nitrila, e poliacrilato de mentila 13, a partir da reação de polimerização radicalar dos respectivos monômeros $\mathbf{7 a}$ e $\mathbf{9}$.

Nas Tabelas 1 e 2 estão apresentados os dados de rendimentos químicos, massas molares, polidispersão e as temperaturas de transição para os copolímeros 10a-d, 11 e dos homopolímeros 12 e 13. Os rendimentos dos copolímeros (Tabela 1) obtidos da reação de copolimerização do monômero isoxazolínico e do mentila foram de moderado a baixo. Os menores rendimentos estão relacionados com os monômeros contendo os grupos polares nitrila e carboxila presentes em 10a e 10d, respectivamente. O maior rendimento foi observado no copolímero 11, no qual foi utilizada maior proporção do comonômero $\mathbf{7 c}$.

As proporções molares dos comonômeros nos copolímeros 10a e 10c ficaram em 71:29, próximas da proporção dos monômeros adicionados no início da polimerização. No entanto, para o copolímero $10 b$ a proporção molar verificada foi 51:49, indicando maior presença de 9 no copolímero resultante. Para o copolímero 10d a proporção de 85:15 indica um incremento do monômero $7 \mathbf{d}$ no material final. Proporção similar foi também encontrada no copolímero $\mathbf{1 1}$.

Os dados das análises de cromatografia de permeação em gel (GPC) e da calorimetria de varredura diferencial (DSC) dos copolímeros 10a-d, 11 e dos homopolímeros $\mathbf{1 2}$ e $\mathbf{1 3}$ estão descritos na Tabela 2. Os copolímeros apresentaram massa molar média entre 6430 e $13240 \mathrm{~g} / \mathrm{mol}$ e com polidispersão na faixa de 1,2 e 1,8. A distribuição dos pesos molares foi menos homogênea para os copolímeros $10 \mathrm{~b}$ e 10d $(\mathrm{PD}=1,8)$. Os copolímeros 10b, 10c e 10d apresentaram maiores inserções monoméricas na cadeia, enquanto 10a e 11 apresentaram os menores graus de polimerização, considerando-se o grau de polimerização descrito na Tabela 2. Com relação aos homopolímeros 12 e 13, o grau de polimerização encontra-se na faixa observada para os copolímeros.

Nas análises de DSC pode-se observar que o único copolímero amorfo é o $\mathbf{1 0 b}$, com um percentual do comonômero de maior anisotropia de 51\%. Nos outros copolímeros, nas análises de microscopia óptica de luz polarizada das amostras, as quais estavam confinadas em duas lâminas de vidro, não foi constatada a presença de texturas típicas relacionadas com mesofases líquido-cristalinas. No entanto, foi observado que alguns domínios das amostras apresentaram uma fraca birrefringência óptica. Apesar da ausência de texturas típicas líquido-cristalinos, a magnitude das entalpias encontradas na transição para o líquido isotrópico, nos valores de 10,0 a 18,0 J/g, nos possibilita inferir que os copolímeros 10a, 10c e 10d possuem alguma organização estrutural responsável pela birrefringência observada. ${ }^{19,20}$ O copolímero 11 apresentou uma entalpia na transição para o estado isotrópico de 4,0 J/g, indicando um caráter amorfo mais acentuado, sem a respectiva correlação com mesofases nemáticas. ${ }^{21}$

O comportamento observado neste trabalho difere dos resultados de poliacrilatos isoxazolínicos diarilssubstituídos publicados por Passo e colaboradores, ${ }^{17}$ os quais apresentaram comportamento líquido-cristalino estável do tipo SmA. Notadamente, a indução de mesofases no material polimérico depende da presença de um segundo substituinte aromático na posição 5 do anel heterocíclico, mesmo que para 10a e 10d o substituinte terminal ligado ao átomo de carbono 5 do anel seja um grupo ciano e carboxílico, respectivamente. Além da reduzida anisotropia de forma do componente em maior quantidade, é possível verificar que a presença do comonômero 9 não favorece a formação de estruturas líquido-cristalinas para os copolímeros 10a-d,

Tabela 2. Dados da análise de GPC e DSC dos copolímeros 10a-d e 11 e dos homopolímeros 12 e 13

\begin{tabular}{|c|c|c|c|c|c|c|c|}
\hline Copolímeros & Mn & Mw & PD & GP & $\operatorname{Tg}\left({ }^{\circ} \mathrm{C}\right)$ & $\operatorname{Tm}\left({ }^{\circ} \mathrm{C}\right)$ & $\Delta \mathrm{H}(\mathrm{J} / \mathrm{g})$ \\
\hline $10 \mathbf{a}$ & 6430 & 8150 & 1,3 & 18 & $-6,0$ & 36,2 & 18,0 \\
\hline $10 \mathrm{~b}$ & 10150 & 18370 & 1,8 & 29 & 4,5 & $\mathrm{a}$ & - \\
\hline $10 \mathrm{c}$ & 12800 & 18510 & 1,5 & 30 & 5,6 & 47,0 & 16,0 \\
\hline 10d & 13240 & 24200 & 1,8 & 33 & 31,0 & 63,0 & 10,0 \\
\hline 11 & 8840 & 10500 & 1,2 & 20 & $-7,0$ & 19,0 & 4,0 \\
\hline \multicolumn{8}{|l|}{ Homopolímeros } \\
\hline 12 & 7010 & 8670 & 1,2 & 17 & 6,2 & a & - \\
\hline 13 & 5960 & 7990 & 1,3 & 29 & 63,0 & $\mathrm{a}$ & - \\
\hline
\end{tabular}

Mn - Massa molar média em g/mol; Mw - Massa molar média por peso em g/mol; PD - Polidispersão e GP - Grau de polimerização. ${ }^{a}$ material amorfo. Tg Temperatura de transição vítrea; Tm - Temperatura de fusão; $\Delta \mathrm{H}$ - variação da entalpia em J/g. 
obtidos na proporção de 70:30. O incremento do monômero 7c na preparação do copolímero 11 (obtido da mistura mol:mol de 7c:9 em 90:10) não foi suficiente para provocar o surgimento de mesofases líquido-cristalinas. Dos resultados obtidos pode-se concluir que a anisotropia geométrica, obtida da razão comprimento:largura $(l / d)$ dos monômeros 7a-d, não é suficiente para manter a ordem orientacional ou posicional necessária para o surgimento das mesofases líquido-cristalinas. O eixo longitudinal $l$ nessa série de poliacrilatos não é suficientemente longo e, assim, a auto-organização molecular é desfavorecida. Adicionalmente, o comonômero 9 tem efeito estérico muito forte nas vizinhas da cadeia polimérica, contribuindo negativamente no empacotamento molecular do grupo rígido de maior anisotropia.

Da mesma forma que os copolímeros 10a-d e 11, os homopolímeros contendo o grupo nitrila terminal 12 e o poliacrilato derivado da mentila 13 não apresentaram comportamento mesomórfico. A presença do grupo polar ciano ligado ao carbono $\mathrm{C}_{5}$ do anel isoxazolina em 12 não foi suficiente para induzir algum comportamento mesomórfico, apesar do momento dipolar do grupo ciano estar localizado no eixo molecular longitudinal. ${ }^{22}$

A ausência de mesofases nos copolímeros sintetizados preclude uma análise da influência da quiralidade do comonômero 9, através das análises das texturas das mesofases quirais. Através dos valores da rotação óptica na faixa de -7 a $-17^{\circ}$ dos copolímeros pode-se afirmar que ocorreu uma diluição deste monômero quiral ao longo da cadeia polimérica dos copolímeros finais.

Em relação à transição vítrea $(\mathrm{Tg})$, observou-se que os pares de copolímeros 10a- 11 e 10b-c apresentaram transições vítreas semelhantes, enquanto que o copolímero 10d apresentou um aumento na $\mathrm{Tg}$.

No copolímero 11, onde a proporção molar foi de 86:14 entre os monômeros 7c e 9, observou-se que a transição vítrea diminuiu $12,6^{\circ} \mathrm{C}$ em relação ao copolímero $10 \mathbf{c}$, que possui os mesmos grupos laterais, porém na proporção 71:29. Esse resultado nos possibilita concluir que a presença do monômero 9 no copolímero determina uma maior restrição conformacional do material amorfo aumentando, assim, a temperatura de transição vítrea.

Os homopolímeros 12 e $\mathbf{1 3}$ são materiais amorfos com temperatura de transição vítrea 6,2 e $63{ }^{\circ} \mathrm{C}$, respectivamente. As diferenças das temperaturas de transição vítrea desses dois homopolímeros estão correlacionadas com a mobilidade da cadeia poliacrílica, a qual, por sua vez, depende da natureza dos grupos ligados lateralmente. ${ }^{10} \mathrm{O}$ argumento do efeito estérico do grupo mentila também se manifesta através da temperatura de transição vítrea maior do homopolímero 13 sobre o homopolímero 12. De outro modo, pode-se dizer que os movimentos rotacionais que ocorrem em segmentos, em partes da cadeia carbônica, localizados no interior ou na periferia da cadeia polimérica, mas não na sua totalidade, são inibidos quando na presença de substituintes mais volumosos, que demandam um volume excluído maior. O incremento encontrado da Tg para o homopolímero 13, de alguma forma, se relacionada com os resultados publicados, nos quais polímeros derivados de ésteres metacrílicos apresentam em geral Tg maiores do que os correspondentes poliacrilatos. ${ }^{23}$

Foi feita análise de difração de raio-X em pó para o composto 10c, com o objetivo de investigar a organização molecular do copolímero quando submetido a ciclos de aquecimento e resfriamento (Figura 3). Foram obtidos três difratogramas de raio-X em 30,45 e $80{ }^{\circ} \mathrm{C}$, que correspondem às temperaturas das fases vítrea, parcialmente cristalina e isotrópica. Os espectros obtidos nessas três temperaturas mostram apenas um sinal largo e difuso na região de alto ângulo, enquanto que na região de baixo ângulo, $2 \theta$, não foram observados picos intensos e na proporção de números inteiros, que estariam relacionados com as reflexões internas da radiação de raio- $\mathrm{X}$ relacionados com ordem esmécticas. Do mesmo modo, não foi observado pico difuso que se associaria à ordem orientacional de mesofase nemática. A 80 ${ }^{\circ} \mathrm{C}$, na região de alto ângulo, observa-se um pico difuso em $2 \theta=$ $19,5^{\circ}$ com espaçamento da ordem de $4,5 \AA$, o qual é característico de fases líquidas isotrópicas e representa a ordem do estado líquido isotrópico - corresponde aproximadamente à distância média entre uma molécula e outra na fase isotrópica. O difratograma obtido a 45 ${ }^{\circ} \mathrm{C}$ apresenta um pico adicional na região de $2 \theta=20,9^{\circ}$. Esse pico persiste em temperaturas menores $\left(30^{\circ} \mathrm{C}\right)$. Através dos difratogramas do copolímero 10c pode-se observar que ocorre um sinal intenso em $2 \theta=21,0^{\circ}$, com o copolímero aquecido acima de sua $\operatorname{Tg}\left(5,6{ }^{\circ} \mathrm{C}\right)$ demonstrando certo nível organizacional na estrutura. Ao aquecer a amostra à temperatura de $45^{\circ} \mathrm{C}$ (Figura 3, curva b) ocorre um alargamento do sinal, indicando perda de organização na estrutura, tendo em vista que o copolímero se encontra a uma temperatura próxima da temperatura de fusão $\left(47,4{ }^{\circ} \mathrm{C}\right.$ ). Acima da Tm, a $80{ }^{\circ} \mathrm{C}$ (Figura 3 , curva c), o sinal se torna bastante difuso, indicando perda total da organização estrutural, devido ao meio isotrópico estabelecido. Os dados assim obtidos de raio-X combinados com as informações da microscopia óptica permitem concluir que a birrefringência observada para esses copolímeros é resultado de algum grau de cristalinidade da amostra, que tem origem no alinhamento parcial dos núcleos duros do monômero $7 \mathbf{c}$ embebido na cadeia do copolímero. O pico de intensidade média observado em $2 \theta=21,0^{\circ}$ e o ombro difuso do lado esquerdo em $2 \theta=20,9^{\circ}$ na Figura 3 , curva b, associado com a ausência de sinal na região de baixo ângulo permite concluir que esta amostra e, por consequência, os demais copolímeros não apresentam comportamento mesomórfico.

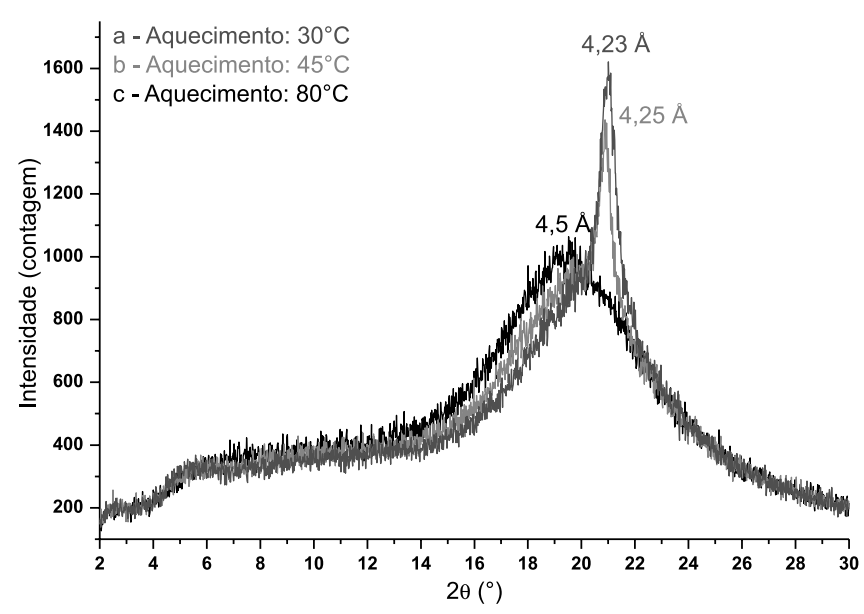

Figura 3. Raio-X do copolímero 10 c a 30,45 e $80^{\circ} \mathrm{C}$

\section{PARTE EXPERIMENTAL}

Os espectros de RMN ${ }^{1} \mathrm{He}$ de ${ }^{13} \mathrm{C}$ foram obtidos no espectrômetro modelo Varian VNMRS - $300 \mathrm{MHz}(7,05 \mathrm{~T})$. As amostras foram preparadas em tubos de $5 \mathrm{~mm}$ de diâmetro em clorofórmio deuterado como solvente e como padrão interno TMS (tetrametilsilano); quando necessário foram adicionadas gotas de dimetilsulfóxido deuterado. Todas as amostras foram analisadas à temperatura ambiente. Os deslocamentos químicos foram dados em ppm. Os espectros de IV foram obtidos após dissolução do produto em THF ou DCM e a sua deposição sobre pastilha de $\mathrm{KBr}$, formando, assim, uma fina camada sobre a superfície da pastilha, utilizando os espectrômetros 300 Galaxy Series. As transições térmicas foram determinadas usando-se um microscópio de luz polarizada Olympus BX-43 acoplado a uma placa de aquecimento FPHT82, controlador Mettler Toledo FP-90 e calorímetro de varredura diferencial (DSC Q-020-TA Instruments), em intervalos de aquecimento e resfriamento de $20^{\circ} \mathrm{C} / \mathrm{min}$. Os 
copolímeros foram caracterizados por cromatografia de permeação em gel (GPC-Waters 150C refractomer). As massas molares obtidas são relativas ao padrão poliestireno.

As reações foram acompanhadas por análise cromatográfica de camada delgada (CCD) com indicador UV e a revelação feita em uma câmara com luz ultravioleta. As purificações foram feitas por lavagem com hexano e recristalização por cromatografia em coluna usando sílica gel 60 Merck 70-230 mesh. Os reagentes utilizados foram p-hidroxibenzaldeído, cloreto de hidroxilamina, acetato de sódio, carbonato de potássio, $N$-clorosuccinimida (NCS), 11-bromo-1-undecanol, ácido acrílico, ácido p-toluenossulfônico ( $p$ TSA), hidroquinona, piridina e 2,2'-azobisisobutironitrila (AIBN) adquiridos das empresas Sigma-Aldrich Inc., Acrós Organics, Merck e grupo Química Industrial Ltda. Os solventes foram tratados de acordo com os métodos tradicionais descritos na literatura.

Seguem abaixo os métodos de preparação e os dados espectrométricos de ${ }^{1} \mathrm{H},{ }^{13} \mathrm{C}$ e infravermelho dos compostos sintetizados.

\section{Síntese e dados do 4-(11-hidroxiundeciloxi)benzaldeído (2)}

Colocou-se em um balão de 150 mL 4-hidroxibenzaldeído (1) (70 $\mathrm{mmol})$, carbonato de potássio $(105 \mathrm{mmol})$ e $252 \mathrm{~mL}$ de acetonitrila sob agitação. Após 15 min adicionou-se gota-a-gota o 11-bromo-1undecanol ( $77 \mathrm{mmol})$ dissolvido em acetonitrila $(20 \mathrm{~mL})$ e deixou-se a reação em refluxo por $72 \mathrm{~h}$. Após o término da reação, filtrou-se a mistura a quente e removeu-se o solvente no rotaevaporador. O sólido obtido foi recristalizado em etanol. Rendimento: $79 \%$; sólido branco amarelado; P.F. $62{ }^{\circ} \mathrm{C}$. RMN ${ }^{1} \mathrm{H}\left(\mathrm{CDCl}_{3}, 300 \mathrm{MHz}\right): \delta 1,5(\mathrm{~m}, 16 \mathrm{H}$, $\left.\left(\mathrm{CH}_{2}\right)_{8}\right) ; 1,8\left(\mathrm{~m}, 2 \mathrm{H}, \mathrm{CH}_{2} \mathrm{CH}_{2} \mathrm{O}\right) ; 3,7\left(\mathrm{t}, 2 \mathrm{H}, \mathrm{CH}_{2} \mathrm{OH}\right) ; 4,1(\mathrm{t}, 2 \mathrm{H}$, $\left.\mathrm{CH}_{2} \mathrm{OAr}\right) ; 7,0$ (d, 2H, Ar, $\left.J=8,7 \mathrm{~Hz}\right) ; 7,8$ (d, 2H, Ar, $J=8,7 \mathrm{~Hz}$ ); 9,9 (s, $1 \mathrm{H}, \mathrm{HCO}) . \mathrm{RMN}^{13} \mathrm{C}\left(\mathrm{CDCl}_{3}, 75 \mathrm{MHz}\right): \delta 25,7 ; 25,9 ; 29,0 ; 29,3$; 29,$4 ; 32,7 ; 63,0 ; 68,3 ; 114,7 ; 129,6 ; 132,0 ; 164,2 ; 190,8$. IV $_{\text {máx }} / \mathrm{cm}^{-1}$ : $3510 ; 2924 ; 2849 ; 1677 ; 1608 ; 1465 ; 1260 ; 1163 ; 1110 ; 839 ; 508$.

\section{Síntese e dados do 4-(11-hidroxiundeciloxi)benzaldeido oxima} (3)

Para a síntese da oxima 3 utilizou-se um balão monotubular de $500 \mathrm{~mL}$ e adicionou-se o aldeído 2 (55,4 $\mathrm{mmol})$, cloridrato de hidroxilamina (155,1 mmol), $216 \mathrm{~mL}$ de etanol e, logo após, acetato de sódio (221,6 mmol) saturado em água. A mistura foi aquecida por 40 min em refluxo; logo após o término da reação, deixou-se a mistura por $24 \mathrm{~h}$ em repouso, sendo filtrada e seca no vácuo, obtendo-se a oxima 3 sem necessidade de purificação. Rendimento: 95\%; sólido branco; P.F. $93{ }^{\circ} \mathrm{C} . \mathrm{RMN}{ }^{1} \mathrm{H}\left(\mathrm{CDCl}_{3} / \mathrm{DMSO}_{\mathrm{d} 6}, 300 \mathrm{MHz}\right): \delta 1,5(\mathrm{~m}$, $\left.16 \mathrm{H},\left(\mathrm{CH}_{2}\right)_{8}\right) ; 1,8\left(\mathrm{~m}, 2 \mathrm{H}, \mathrm{C}_{2} \mathrm{CH}_{2} \mathrm{O}\right) ;$ ); 3,6 (t, 2H, $\left.\mathrm{CH}_{2} \mathrm{OH}\right) ; 4,0(\mathrm{t}$, $2 \mathrm{H}, \mathrm{CH}_{2} \mathrm{OAr}$ ); 6,9 (d, 2H, Ar, $\left.J=8,7 \mathrm{~Hz}\right) ; 7,5$ (d, 2H, Ar, $J=8,7$ $\mathrm{Hz}) ; 8,1$ (s, $1 \mathrm{H}, \mathrm{NOH}) . \mathrm{RMN}^{13} \mathrm{C}\left(\mathrm{CDCl}_{3} / \mathrm{DMSO}_{\mathrm{d} 6}, 75 \mathrm{MHz}\right): \delta 25,5$; 25,$7 ; 28,8 ; 29,0 ; 29,2 ; 29,3 ; 32,5 ; 62,2 ; 67,7 ; 114,3 ; 125,0 ; 127,9$; 148,$5 ; 159,8$. IV $v_{\text {máx }} / \mathrm{cm}^{-1}: 3473 ; 3274 ; 2925 ; 2854 ; 1609 ; 1509$; $1467 ; 1305 ; 1255 ; 1174 ; 1042 ; 951$.

\section{Síntese e dados do 3-[4-(11-hidroxiundeciloxi)fenil]-4,5- di-hidroisoxazol-5-carbonitrila (5a)}

\section{Procedimento de síntese representativo para os monômeros 5a-d}

Utilizou-se um balão de Schlenck de $250 \mathrm{~mL}$ em atmosfera inerte, sob banho de gelo; colocaram-se $35 \mathrm{~mL}$ de DCM destilado e seco, ácido acrílico (10 mmol), NCS (15 mmol) e piridina destilada (15 mmol). Dissolveu-se a oxima 3 em $15 \mathrm{~mL}$ de DCM, que foi adicionada gota-a-gota, lentamente no balão; logo após a adição total, retirou-se o banho de gelo e aqueceu-se até a temperatura ambiente por 4 h. Foi removido o solvente no rotavapor e adicionados $50 \mathrm{~mL}$ de éter etílico. Logo após, lavou-se a mistura com as soluções de $\mathrm{HCl}$ $1 \mathrm{M}(3$ x $20 \mathrm{~mL}), \mathrm{NaHCO}_{3}$ saturada (1 x $\left.15 \mathrm{~mL}\right)$, água destilada (1 x $15 \mathrm{~mL})$ e, finalmente, $\mathrm{NaCl}$ saturado $(1$ x $20 \mathrm{~mL})$. À solução orgânica adicionou-se $\mathrm{Na}_{2} \mathrm{SO}_{4}$ anidro para a retirada de água do meio. A mistura foi filtrada, removido o solvente no rotavapor e o intermediário 5a foi purificado por recristalização em etanol/água. Rendimento: $58 \%$; sólido amarelo; P.F. $92{ }^{\circ} \mathrm{C}$. $\mathrm{RMN}{ }^{1} \mathrm{H}\left(\mathrm{CDCl}_{3}, 300 \mathrm{MHz}\right): \delta 1,4$ (m, 16H, $\left.\left(\mathrm{CH}_{2}\right)_{8}\right) ; 1,8\left(\mathrm{~m}, 2 \mathrm{H}, \mathrm{CH}_{2} \mathrm{CH}_{2} \mathrm{O}\right) ;$ ); 3,6 (t, 2H, $\left.\mathrm{CH}_{2} \mathrm{OH}\right)$; 3,7 (m, 2H, $\underline{\mathrm{HHCH}}$ ); 4,0 (t, 2H, $\left.\underline{\mathrm{H}}_{2} \mathrm{OAr}\right) ; 5,3$ (dd, 1H, CHHC$\underline{\mathrm{H}}$, $\left.{ }^{3} J_{\text {trans }}=6,5 \mathrm{~Hz},{ }^{3} J_{\text {cis }}=10,2 \mathrm{~Hz}\right) ; 6,9(\mathrm{~d}, 2 \mathrm{H}, \mathrm{Ar}, J=8,8 \mathrm{~Hz}) ; 7,6(\mathrm{~d}$, $2 \mathrm{H}, \mathrm{Ar}, J=8,8 \mathrm{~Hz}) . \mathrm{RMN}{ }^{13} \mathrm{C}\left(\mathrm{CDCl}_{3}, 75 \mathrm{MHz}\right): \delta 25,6 ; 25,8 ; 28,9$; 29,$2 ; 29,3 ; 29,4 ; 32,6 ; 41,2 ; 62,7 ; 66,2,68,1 ; 114,8 ; 117,2 ; 119,3$; 128,$6 ; 155,9 ; 161,3$. IV $v_{\text {máx }} / \mathrm{cm}^{-1}: 3361 ; 2914 ; 2846 ; 1611 ; 1520$; $1469 ; 1257 ; 1065 ; 832$.

\section{3-[4-(11-hidroxiundeciloxi)fenil]-4,5-di-hidroisoxazol-5-carboxi- lato de butila $\mathbf{( 5 \mathbf { b } )}$}

Rendimento: $61 \%$; sólido bege; P.F. $70-72{ }^{\circ} \mathrm{C}$. $\mathrm{RMN}{ }^{1} \mathrm{H}\left(\mathrm{CDCl}_{3} /\right.$ $\left.\mathrm{DMSO}_{\mathrm{d} 6}, 300 \mathrm{MHz}\right): \delta 0,9\left(\mathrm{t}, 3 \mathrm{H}, \mathrm{CH}_{3}\right) ; 1,6\left(\mathrm{~m}, 22 \mathrm{H},\left(\mathrm{CH}_{2}\right)_{11}\right)$; 3,6 (m, 4H, CHㅐ,$\left.\underline{\mathrm{H}}_{2} \mathrm{OH}\right) ; 4,0$ (t, 2H, $\left.\underline{\mathrm{H}}_{2} \mathrm{OAr}\right) ; 4,2$ (t, 2H, $\left.\underline{\mathrm{CH}}_{2} \mathrm{OCO}\right) ; 5,1$ (md, $\left.1 \mathrm{H}, \mathrm{CHHC} \underline{\mathrm{H}}\right) ; 6,9$ (d, 2H, Ar, $\left.J=8,8 \mathrm{~Hz}\right)$; $7,6$ (d, $2 \mathrm{H}, \mathrm{Ar}, J=8,8 \mathrm{~Hz})$. RMN ${ }^{13} \mathrm{C}\left(\mathrm{CDCl}_{3}, 75 \mathrm{MHz}\right): \delta 13,6$; 18,$9 ; 25,6 ; 25,9 ; 29,0 ; 29,2 ; 29,3 ; 29,4 ; 29,5 ; 30,4 ; 32,6 ; 39,0$; 62,$8 ; 65,7 ; 68,0 ; 114,6 ; 120,7 ; 128,4 ; 155,5 ; 160,8 ; 177,4$. IV $v_{\text {máx. }} / \mathrm{cm}^{-1}: 3421 ; 3353 ; 2913 ; 2846 ; 1743 ; 1614 ; 1518 ; 1467 ; 1355$; $1254 ; 1203 ; 1061 ; 889 ; 829$.

\section{3-[4-(11-hidroxiundeciloxi)fenil]-4,5-di-hidroisoxazol-5-carboxi-} lato de hexila $(\mathbf{5 c})$

Rendimento: $68 \%$; sólido bege; P.F. $71-73{ }^{\circ} \mathrm{C} . \mathrm{RMN}{ }^{1} \mathrm{H}\left(\mathrm{CDCl}_{3}, 300\right.$ $\mathrm{MHz}): \delta 0,9\left(\mathrm{t}, 3 \mathrm{H}, \mathrm{CH}_{3}\right) ; 1,5\left(\mathrm{~m}, 28 \mathrm{H},\left(\mathrm{CH}_{2}\right)_{14}\right) ; 3,6(\mathrm{~m}, 4 \mathrm{H}, \mathrm{CHHCH}$,

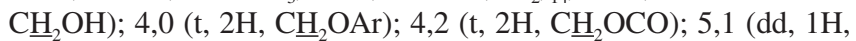
$\left.\mathrm{CHHC} \underline{\mathrm{H}},{ }^{3} J_{\text {trans }}=8,4 \mathrm{~Hz},{ }^{3} J_{\text {cis }}=9,8 \mathrm{~Hz}\right) ; 6,9(\mathrm{~d}, 2 \mathrm{H}, \mathrm{Ar}, J=8,8 \mathrm{~Hz})$; $7,6$ (d, $2 \mathrm{H}, \mathrm{Ar}, J=8,8 \mathrm{~Hz}) . \mathrm{RMN}{ }^{13} \mathrm{C}\left(\mathrm{CDCl}_{3}, 75 \mathrm{MHz}\right): \delta 13,9 ; 22,4$; 25,$3 ; 25,7 ; 25,9 ; 28,3 ; 29,0 ; 29,3 ; 29,4,29,5 ; 31,3 ; 32,7 ; 39,1 ; 62,9$; 66,$0 ; 68,0 ; 77,7 ; 114,6 ; 120,7 ; 128,3 ; 155,5 ; 160,8 ; 170,4 . I V v_{\text {máx }} /$ $\mathrm{cm}^{-1}: 3447 ; 2917 ; 2846 ; 1749 ; 1607 ; 1515 ; 1466 ; 1352 ; 1259 ; 1213$; $1174 ; 1064 ; 890 ; 834$.

Ácido 3-[4-(11-hidroxiundeciloxi)fenil]-4,5-di-hidroisoxazol-5- carboxílico $(\mathbf{5 d})$

Rendimento: 68\%; sólido amarelo; P.F. $111-113{ }^{\circ} \mathrm{C}$. RMN ${ }^{1} \mathrm{H}$ $\left(\mathrm{CDCl}_{3} / \mathrm{DMSO}_{\mathrm{d} 6}, 300 \mathrm{MHz}\right): \delta 1,2\left(\mathrm{~m}, 16 \mathrm{H},\left(\mathrm{CH}_{2}\right)_{8}\right) ; 1,6(\mathrm{~m}, 2 \mathrm{H}$, $\left.\mathrm{CH}_{2} \mathrm{CH}_{2} \mathrm{O}\right) ; 3,4$ (m, 4H, CHHCH, $\left.\underline{\mathrm{H}}_{2} \mathrm{OH}\right) ; 3,8$ (t, 2H, $\left.\mathrm{CH}_{2} \mathrm{O}\right) ; 4,9$ $(\mathrm{m}, 1 \mathrm{H}, \mathrm{CHHC} \underline{\mathrm{H}}) ; 6,7$ (d, 2H, Ar, $J=8,8 \mathrm{~Hz}) ; 7,4$ (d, 2H, Ar, $J=8,8$ $\mathrm{Hz})$. $\mathrm{RMN}{ }^{13} \mathrm{C}\left(\mathrm{CDCl}_{3} / \mathrm{DMSO}_{\mathrm{d} 6}, 75 \mathrm{MHz}\right): \delta 25,4 ; 25,5 ; 28,7 ; 28,9$; 29,$0 ; 29,1 ; 32,4 ; 38,8 ; 62,0 ; 67,7 ; 77,2 ; 114,2 ; 120,5 ; 128,0 ; 155,2$; 160,$4 ; 172,0$. IV $v_{\text {max }} / \mathrm{cm}^{-1}: 3283 ; 2914 ; 2846 ; 1726 ; 1611 ; 1515$; $1466 ; 1253 ; 1278 ; 1168 ; 894 ; 830 ; 534$.

\section{Síntese e dados do acrilato de 11-[4-(5-ciano-4,5-di-hidroisoxa-} zol-3-il)feniloxi] undecila (7a)

\section{Procedimento de síntese representativo para os monômeros $\mathbf{7 a - d}$ e 9}

O monômero 7a foi produzido colocando-se em um balão, acoplado a um sistema Dean-Stark, o intermediário $\mathbf{5 a}$ (5,4 mmol), ácido acrílico (40 mmol) em $50 \mathrm{~mL}$ de benzeno, adicionou-se ácido p-toluenossulfônico ( $1 \mathrm{mmol}$ ) e hidroquinona (5,4 mmol). O sistema foi colocado em refluxo por $4 \mathrm{~h}$. Após esse tempo, a solução foi filtrada a quente e o filtrado concentrado no rotavapor. O monômero $7 \mathbf{a}$ foi purificado por recristalização em isopropanol. Rendimento: 42\%; sólido bege; P.F. $74-77{ }^{\circ} \mathrm{C}$. RMN ${ }^{1} \mathrm{H}\left(\mathrm{CDCl}_{3}, 300 \mathrm{MHz}\right): \delta 1,3(\mathrm{~m}$, 
$\left.14 \mathrm{H},\left(\mathrm{CH}_{2}\right)_{7}\right) ; 1,7$ (q, 2H, $\left.\underline{\mathrm{H}}_{2} \mathrm{CH}_{2} \mathrm{O}\right) ; 1,8$ (q, 2H, $\left.\underline{\mathrm{C}}_{2} \mathrm{CH}_{2} \mathrm{OCO}\right) ; 3,7$ (m, 2H, $\underline{\mathrm{HHCH}}$ ); 4,0 (t, 2H, $\left.\mathrm{CH}_{2} \mathrm{O}\right) ; 4,1$ (t, 2H, $\left.\mathrm{CH}_{2} \mathrm{OCO}\right) ; 5,3$ (dd, $\left.1 \mathrm{H}, \mathrm{CHHC} \underline{\mathrm{H}},{ }^{3} J_{\text {trans }}=6,7 \mathrm{~Hz},{ }^{3} \mathrm{~J}_{\text {cis }}=10,1 \mathrm{~Hz}\right) ; 5,8\left(\mathrm{dd}, 1 \mathrm{H}, \mathrm{CH}=\mathrm{CH}_{2}\right.$, $\left.{ }^{2} J_{\text {gem }}=1,6 \mathrm{~Hz},{ }^{3} J_{\text {cis }}=10,4 \mathrm{~Hz}\right) ; 6,1\left(\mathrm{dd}, 1 \mathrm{H}, \mathrm{CH}=\mathrm{CH}_{2},{ }^{3} J_{\text {cis }}=10,4 \mathrm{~Hz}\right.$ $\left.{ }^{3} J_{\text {trans }}=17,3 \mathrm{~Hz}\right) ; 6,4\left(\mathrm{dd}, 1 \mathrm{H}, \mathrm{CH}=\mathrm{CH}_{2},{ }^{2} J_{\text {gem }}=1,6 \mathrm{~Hz},{ }^{3} J_{\text {trans }}=17,3\right.$ $\mathrm{Hz}) ; 6,9$ (d, 2H, Ar, $J=8,9 \mathrm{~Hz}) ; 7,6$ (d, 2H, Ar, $J=8,9 \mathrm{~Hz})$. RMN ${ }^{13} \mathrm{C}\left(\mathrm{CDCl}_{3}, 75 \mathrm{MHz}\right): \delta 25,9 ; 28,5 ; 29,0 ; 29,1 ; 29,2 ; 29,4 ; 41,3 ; 64,6$; 66,$2 ; 68,1 ; 114,8 ; 117,2 ; 119,4 ; 128,6 ; 130,4 ; 155,8 ; 161,4 ; 166,3$. IV $v_{\text {máx. }} / \mathrm{cm}^{-1}: 2916 ; 2850 ; 2360 ; 1724 ; 1608 ; 1517 ; 1477 ; 1409 ; 1359$; $1301 ; 1255 ; 1201 ; 1141 ; 995 ; 833 ; 648 ; 547 ; 418$.

3-[4-(11-Acriloiloxi)undeciloxi)fenil]-4,5-di-hidroisoxazol-5-carboxilato de butila $\mathbf{( 7 b )}$

Rendimento: $33 \%$; sólido amarelo; P.F. $111-112{ }^{\circ} \mathrm{C}$. RMN ${ }^{1} \mathrm{H}$ $\left(\mathrm{CDCl}_{3}, 300 \mathrm{MHz}\right): \delta 0,9\left(\mathrm{t}, 3 \mathrm{H}, \mathrm{CH}_{3}\right) ; 1,3\left(\mathrm{~m}, 18 \mathrm{H},\left(\mathrm{CH}_{2}\right)_{9}\right) ; 1,7$ $\left(\mathrm{m}, 4 \mathrm{H},\left(\mathrm{CH}_{2}\right)_{2}\right) ; 3,6(\mathrm{~m}, 2 \mathrm{H}, \mathrm{CHHCH}) ; 4,0\left(\mathrm{t}, 2 \mathrm{H}, \mathrm{CH}_{2} \mathrm{O}\right) ; 4,2(\mathrm{~m}$, $\left.4 \mathrm{H}, \mathrm{CH}_{2} \mathrm{O}, \mathrm{CH}_{2} \mathrm{OCO}\right) ; 5,1\left(\mathrm{dd}, 1 \mathrm{H}, \mathrm{CHHC} \underline{\mathrm{H}},{ }^{3} J_{\text {trans }}=8,4 \mathrm{~Hz},{ }^{3} J_{\text {cis }}\right.$ $=9,7 \mathrm{~Hz}) ; 5,8\left(\mathrm{dd}, 1 \mathrm{H}, \mathrm{CH}=\mathrm{CH}_{2},{ }^{2} J_{\text {gem }}=1,6 \mathrm{~Hz},{ }^{3} J_{\text {cis }}=10,4 \mathrm{~Hz}\right)$; $6,1\left(\mathrm{dd}, 1 \mathrm{H}, \mathrm{CH}=\mathrm{CH}_{2},{ }^{3} J_{\text {cis }}=10,4 \mathrm{~Hz}{ }^{3} J_{\text {trans }}=17,3 \mathrm{~Hz}\right) ; 6,4(\mathrm{dd}$, $\left.1 \mathrm{H}, \mathrm{CH}=\mathrm{CH}_{2},{ }^{2} J_{\text {gem }}=1,6 \mathrm{~Hz},{ }^{3} J_{\text {trans }}=17,3 \mathrm{~Hz}\right) ; 6,9(\mathrm{~d}, 2 \mathrm{H}, \mathrm{Ar}, J=$ $8,8 \mathrm{~Hz}) ; 7,6(\mathrm{~d}, 2 \mathrm{H}, \mathrm{Ar}, J=8,8 \mathrm{~Hz}) . \mathrm{RMN}{ }^{13} \mathrm{C}\left(\mathrm{CDCl}_{3}, 75 \mathrm{MHz}\right)$ : 13,$6 ; 18,9 ; 25,6 ; 25,8 ; 25,9 ; 28,3 ; 28,5 ; 29,0 ; 29,1 ; 29,2 ; 29,3 ; 29,4$; 30,$4 ; 39,0 ; 64,6 ; 65,7 ; 65,9 ; 68,0 ; 77,7 ; 114,6 ; 120,7 ; 128,3 ; 128,5$; 130,$4 ; 155,5 ; 160,8 ; 166,2 ; 170,4$. IV $v_{\text {máx. }} / \mathrm{cm}^{-1}: 2918 ; 2852 ; 2360$; 1716; 1608; 1516; 1469; 1408; 1357; 1255; 1995; 1160; 983; 896; $833 ; 736 ; 646 ; 545$.

3-[4-(11-Acriloiloxiundecil)oxifenil]-4,5-di-hidroisoxazol-5-carboxilato de hexila $(\mathbf{7 c})$

Rendimento: 28\%; sólido amarelo; P.F. $71{ }^{\circ} \mathrm{C}$. $\mathrm{RMN}{ }^{1} \mathrm{H}\left(\mathrm{CDCl}_{3}\right.$, $300 \mathrm{MHz}): \delta 1,0\left(\mathrm{t}, 3 \mathrm{H}, \mathrm{CH}_{3}\right) ; 1,3\left(\mathrm{~m}, 20 \mathrm{H},\left(\mathrm{CH}_{2}\right)_{10}\right) ; 1,7(\mathrm{~m}, 6 \mathrm{H}$, $\left.\left(\mathrm{CH}_{2}\right)_{3}\right) ; 3,6(\mathrm{~m}, 2 \mathrm{H}, \mathrm{CHHCH}) ; 4,0\left(\mathrm{t}, 2 \mathrm{H}, \mathrm{CH}_{2} \mathrm{O}\right) ; 4,2(\mathrm{~m}, 4 \mathrm{H}$, $\mathrm{CH}_{2} \mathrm{O}, \mathrm{CH}_{2} \mathrm{OCO}$ ); 5,1 (dd, $1 \mathrm{H}, \mathrm{CHHC} \underline{\mathrm{H}},{ }^{3} J_{\text {trans }}=8,5 \mathrm{~Hz},{ }^{3} J_{\text {cis }}=10,0$ $\mathrm{Hz}) ; 5,8\left(\mathrm{dd}, 1 \mathrm{H}, \mathrm{CH}=\mathrm{CH}_{2},{ }^{2} J_{\text {gem }}=1,6 \mathrm{~Hz},{ }^{3} \mathrm{~J}_{\text {cis }}=10,4 \mathrm{~Hz}\right) ; 6,1(\mathrm{dd}$, $1 \mathrm{H}, \mathrm{CH}=\mathrm{CH}_{2},{ }^{3} J_{\text {cis }}=10,4 \mathrm{~Hz}^{3}{ }^{3}$ trans $\left.=17,3 \mathrm{~Hz}\right) ; 6,4\left(\mathrm{dd}, 1 \mathrm{H}, \mathrm{CH}=\mathrm{CH}_{2}\right.$, $\left.{ }^{2} J_{\text {gem }}=1,6 \mathrm{~Hz},{ }^{3} J_{\text {trans }}=17,3 \mathrm{~Hz}\right) ; 6,9(\mathrm{~d}, 2 \mathrm{H}, \mathrm{Ar}, J=8,8 \mathrm{~Hz}) ; 7,6(\mathrm{~d}$, $2 \mathrm{H}, \mathrm{Ar}, J=8,8 \mathrm{~Hz}) . \mathrm{RMN}{ }^{13} \mathrm{C}\left(\mathrm{CDCl}_{3}, 75 \mathrm{MHz}\right) \delta 13,9 ; 22,4 ; 25,8$; 25,$9 ; 28,3 ; 28,4 ; 28,5 ; 29,1 ; 29,2 ; 29,3 ; 29,4 ; 31,3 ; 39,1 ; 64,7 ; 66,0$; 68,$2 ; 77,9 ; 114,6,120,7 ; 128,4 ; 128,5 ; 155,5 ; 160,9 ; 164,7 ; 170,4$. IV $v_{\text {máx }} / \mathrm{cm}^{-1}: 2924 ; 2852 ; 2360 ; 1739 ; 1608 ; 1516 ; 1467 ; 1355$; 1255; 1997; 833.

Ácido 3-[4-(11-acriloiloxi)undeciloxi]fenil-4,5-di-hidroisoxazol-5- arboxilico $\mathbf{( 7 d )}$

Rendimento: 44\%; sólido bege escuro; P.F. $103-104{ }^{\circ} \mathrm{C}$. RMN ${ }^{1} \mathrm{H}$ $\left(\mathrm{CDCl}_{3}, 300 \mathrm{MHz}\right): \delta 1,3\left(\mathrm{~m}, 14 \mathrm{H},\left(\mathrm{CH}_{2}\right)_{7}\right) ; 1,7\left(\mathrm{~m}, 4 \mathrm{H}, \mathrm{C}_{2} \mathrm{CH}_{2} \mathrm{O}\right.$, $\left.\mathrm{C}_{2} \mathrm{CH}_{2} \mathrm{OCO}\right) ; 3,6(\mathrm{~m}, 2 \mathrm{H}, \mathrm{CHHCH}) ; 4,0\left(\mathrm{t}, 2 \mathrm{H}, \mathrm{CH}_{2} \mathrm{O}\right) ; 4,2(\mathrm{~m}$, $\left.4 \mathrm{H}, \mathrm{CH}_{2} \mathrm{O}, \mathrm{CH}_{2} \mathrm{OCO}\right) ; 5,1\left(\mathrm{dd}, 1 \mathrm{H}, \mathrm{CHH}-\mathrm{C} \underline{\mathrm{H}},{ }^{3} J_{\text {trans }}=8,1 \mathrm{~Hz},{ }^{3} J_{\text {cis }}\right.$ $=10,1 \mathrm{~Hz}) ; 5,8\left(\mathrm{dd}, 1 \mathrm{H}, \mathrm{CH}=\mathrm{CH}_{2},{ }^{2} J_{\text {gem }}=1,6 \mathrm{~Hz},{ }^{3} J_{\text {cis }}=10,4 \mathrm{~Hz}\right)$; $6,1\left(\mathrm{dd}, 1 \mathrm{H}, \mathrm{CH}=\mathrm{CH}_{2},{ }^{3} J_{\text {cis }}=10,4 \mathrm{~Hz}{ }^{3} J_{\text {trans }}=17,3 \mathrm{~Hz}\right) ; 6,4(\mathrm{dd}, 1 \mathrm{H}$, $\left.\mathrm{CH}=\mathrm{CH}_{2},{ }^{2} J_{\text {gem }}=1,6 \mathrm{~Hz},{ }^{3} J_{\text {trans }}=17,3 \mathrm{~Hz}\right) ; 6,9(\mathrm{~d}, 2 \mathrm{H}, \mathrm{Ar}, J=8,8 \mathrm{~Hz})$; $7,6(\mathrm{~d}, 2 \mathrm{H}, \mathrm{Ar}, J=8,8 \mathrm{~Hz}) . \mathrm{RMN}^{13} \mathrm{C}\left(\mathrm{CDCl}_{3} / \mathrm{DMSO}_{\mathrm{d} 6}, 75 \mathrm{MHz}\right): \delta$ 25,$6 ; 25,9 ; 28,3 ; 29,0 ; 29,1 ; 29,2 ; 29,3 ; 29,4 ; 39,0 ; 66,0 ; 68,0 ; 77,8$; $114,6,120,7 ; 128,4 ; 128,5 ; 155,5 ; 160,8 ; 164,6 ; 170,4 . \mathrm{IV} \mathrm{v}_{\text {máx }} / \mathrm{cm}^{-1}$ : $2922 ; 2852 ; 1732 ; 1608 ; 1516 ; 1471 ; 1355 ; 1257 ; 1201 ; 1041 ; 891$; $831 ; 736 ; 545$.

Acrilato de (1R,2R,5R)-2-isopropil-5-metilciclo-hexila (9) Dados espectroscópicos de acordo com a ref. 24. Rendimento: $65 \%$; líquido incolor; $[\alpha]_{\mathrm{D}}^{20}=-77^{\circ}\left(10, \mathrm{CH}_{2} \mathrm{Cl}_{2}\right)$. RMN ${ }^{1} \mathrm{H}\left(\mathrm{CDCl}_{3}, 300\right.$ $\mathrm{MHz}): \delta 0,7(\mathrm{~m}, 4 \mathrm{H}) ; 0,9(\mathrm{~m}, 8 \mathrm{H}) ; 1,4(\mathrm{~m}, 2 \mathrm{H}) ; 1,7(\mathrm{~m}, 2 \mathrm{H}) ; 1,8(\mathrm{~m}$, $1 \mathrm{H}) ; 2,0(\mathrm{~m}, 1 \mathrm{H}) ; 4,8(\mathrm{dt}, 1 \mathrm{H}, \mathrm{C} \underline{\mathrm{HOCO}}, J=4,4 \mathrm{~Hz}, J=10,9 \mathrm{~Hz})$; $5,8\left(\mathrm{dd}, 1 \mathrm{H}, \mathrm{CH}=\mathrm{CH}_{2},{ }^{2} J_{\mathrm{gem}}=1,6 \mathrm{~Hz},{ }^{3} J_{\text {cis }}=10,4 \mathrm{~Hz}\right) ; 6,1(\mathrm{dd}, 1 \mathrm{H}$,
$\left.\mathrm{CH}=\mathrm{CH}_{2},{ }^{3} J_{\text {cis }}=10,4 \mathrm{~Hz}{ }^{3} J_{\text {trans }}=17,3 \mathrm{~Hz}\right) ; 6,4\left(\mathrm{dd}, 1 \mathrm{H}, \mathrm{CH}=\mathrm{CH}_{2}\right.$, $\left.{ }^{2} J_{\text {gem }}=1,6 \mathrm{~Hz},{ }^{3} J_{\text {trans }}=17,3 \mathrm{~Hz}\right) . \mathrm{RMN}^{13} \mathrm{C}\left(\mathrm{CDCl}_{3}, 75 \mathrm{MHz}\right): \delta 16,3$; 20,$7 ; 22,0 ; 23,5 ; 26,3 ; 31,3 ; 34,2 ; 40,8 ; 47,0 ; 74,2 ; 129,0 ; 130,1$; 165,7 . IV $v_{\text {max }} / \mathrm{cm}^{-1}: 2953 ; 2868 ; 1722 ; 1456 ; 1406 ; 1294 ; 1269$; 1197; 1047; 983; 808 .

Síntese e dados do poliacrilato de 11-[4-(5-ciano-4,5-di-hidroisoxazol-3-il)fenoxi]undecila-coacrilato de $(1 R, 2 R, 5 R)-2$-isopropil-5-metilciclo-hexila (10a)

Procedimento de síntese representativo para os copolímeros 10a-d na proporção molar 70:30: num balão de Schlenck com tampa, adicionaram-se os monômeros $7 \mathbf{a}(0,82 \mathrm{mmol}, 0,4 \mathrm{~g})$ e $\mathbf{9}(0,35 \mathrm{mmol}$, $0,073 \mathrm{~g}$ ) com $5 \mathrm{~mL}$ de tolueno, destilado e seco, em atmosfera inerte; agitou-se o sistema durante $30 \mathrm{~min}$. Logo após, foi adicionado 5\% em massa de AIBN e o balão foi lacrado. Aqueceu-se à temperatura de $65^{\circ} \mathrm{C}$ por $72 \mathrm{~h}$. Após as $72 \mathrm{~h}$, vaporizou-se o tolueno e o resíduo sólido foi lavado com hexano num extrator de Soxhlet, com o acompanhamento do desaparecimento do sinal dos hidrogênios olefínicos do grupo acrilato por RMN ${ }^{1} \mathrm{H}$, a fim de remover o monômero não polimerizado. Rendimento: $40 \%$; líquido viscoso amarelo; $[\alpha]_{\mathrm{D}}^{20}=$ $-7^{\circ}(10, \mathrm{DCM}) ; \mathrm{Tg}=-6,0{ }^{\circ} \mathrm{C} ; \mathrm{Tm}=36,2{ }^{\circ} \mathrm{C}$.

Poli\{3-[4-(11-acriloiloxiundecil)oxifenil]\}-4,5-di-hidroisoxazol-5-carboxilato de butila coacrilato de $(1 R, 2 R, 5 R)$-2-isopropil-5metilciclo-hexila $\mathbf{( 1 0 b )}$

Rendimento: $54 \%$; líquido viscoso amarelo; $[\alpha]_{\mathrm{D}}^{20}=-9^{\circ}\left(10, \mathrm{CH}_{2} \mathrm{Cl}_{2}\right)$; $\operatorname{Tg}=4,5^{\circ} \mathrm{C}$.

Poli\{3-[4-(11-acriloiloxiundecil)oxifenil]\}-4,5-di-hidroisoxazol-5-carboxilato de hexila-coacrilato de $(1 R, 2 R, 5 R)$-2-isopropil-5metilciclo-hexila $(\mathbf{1 0 c})$

Rendimento: $57 \%$. Sólido amarelo; $[\alpha]_{\mathrm{D}}^{20}=-10^{\circ}\left(10, \mathrm{CH}_{2} \mathrm{Cl}_{2}\right) ; \mathrm{Tg}$ $=5,6{ }^{\circ} \mathrm{C} ; \mathrm{Tm}=47,0{ }^{\circ} \mathrm{C}$.

Poli\{ácido 3-[4-(11-acriloiloxiundeciloxi)fenil]\}-4,5-di-hidroisoxazol-5-carboxilico-coacrilato de (1R,2R,5R)-2-isopropil-5-metilciclohexila (10d)

Rendimento: $29 \%$. Sólido amarelo; $[\alpha]_{\mathrm{D}}^{20}=-17^{\circ}(10, \mathrm{THF}) ; \mathrm{Tg}=$ $31,0{ }^{\circ} \mathrm{C} ; \mathrm{Tm}=63,0{ }^{\circ} \mathrm{C}$.

Síntese e dados do poli\{3-[4-(11-acriloiloxiundecil) oxifenil]\}-4,5-di-hidroisoxazol-5-carboxilato de hexila-coacrilato de $(1 R, 2 R, 5 R)-2$-isopropil-5-metilciclo-hexila (11)

Procedimento semelhante ao realizado para os copolímeros 10a-d com proporção molar de 90:10. Rendimento: 64\%; líquido viscoso amarelo; $[\alpha]_{\mathrm{D}}^{20}=-7^{\circ}\left(10, \mathrm{CH}_{2} \mathrm{Cl}_{2}\right) ; \mathrm{Tg}=-7,0^{\circ} \mathrm{C} ; \mathrm{Tm}=19,0{ }^{\circ} \mathrm{C}$.

\section{CONCLUSÃO}

Foram produzidos quatro novos copolímeros 10a-d em proporções molares de 70:30 dos respectivos monômeros e o copolímero 11 em proporção molar de 90:10, os quais apresentaram birrefringência óptica, porém com ausência de mesofases líquido-cristalinas. Do mesmo modo, foram sintetizados homopolímeros contendo grupos terminais nitrila $\mathbf{1 2}$ e mentila $\mathbf{1 3}$, que não apresentaram comportamento mesomórfico. Nos copolímeros sintetizados 10a-d e 11 não se observou indução quiral de mesofases, em virtude da ausência de mesofases nesses compostos finais. Constatou-se que a substituição por grupos menores na posição 5 do anel isoxazolínico altera a relação l/d associada com a anisometria de forma, inibindo desta maneira o aparecimento de mesofases. 


\section{MATERIAL SUPLEMENTAR}

Está disponível gratuitamente em http://quimicanova.sbq.org.br, na forma de arquivo PDF.

\section{AGRADECIMENTOS}

Ao CNPq através do Edital MCT/CNPq 14/2008 - Universal 2008, Faixa A e do edital PQ 10/2009-Produtividade em Pesquisa. À CAPES via edital PROCAD/CAPES e ao INCT-Catálise pelo apoio financeiro. Ao Instituto de Química - UFRGS pela infraestrutura disponível. A. A. Merlo agradece ao CNPq pela bolsa de pós-doutoramento/2012.

\section{REFERÊNCIAS}

1. Coelho, M. R. G.; Gomes, A. S.; Quim. Nova 2007, 30, 636.

2. Kishi, H.; Kunimitsu, Y.; Imade, J.; Oshita, S.; Morishita, Y.; Asada, M.; Polymer 2011, 52, 760; Yu, H.; Kobayashi, T.; Molecules 2010, 15, 570.

3. Kumar, G.; Nisha, N.; Mageswari, S.; Subramanian, K.; J. Polym. Res. 2011, $18,241$.

4. Jenkins, D. W.; Hudson, S. M.; Chem. Rev. 2001, 101, 3245; Yuan, W.; Li, X.; Gu, S.; Cao, A.; Ren , J.; Polymer 2011, 52, 658.

5. Jiang, X.; Gu, J.; Shen, Y.; Wang, S.; Tian, X.; Desalination 2011, 265, 74.

6. Kiharaa, H.; Kishia, R.; Miuraa, T.; Katob, T.; Ichijo, H.; Polym. Res. 2011, 18, 171 .

7. Echeverria, C.; Lopez, D.; Mijang, C.; Macromolecules 2009, 42, 9118.

8. Kawatsuki, N.; Uchida, E.; Yamamoto, T.; Macromol. Chem. Phys. 2003, 204, 584; Zhou, Y.; Ahn, S.; Lakhman, R. K.; Gopinadhan, M.; Osuji, C. O.; Kasi, R. M.; Macromolecules 2011, 44, 3924; Barmatov, E. B.; Pebalk, D. A.; Barmatova, M. V.; Shibaev, V. P.; Liq. Cryst. 1997, 23 , 447; Barmatov, E. B.; Barmatova, M. V.; Shibaev, V. P.; Colloid Polym. Sci. 1998, 276, 662.

9. Vilela, G. D.; Rosa, R. R.; Schneider, P. H.; Bechtold, I. H.; Eccher, J.; Merlo, A. A.; Tetrahedron Lett. 2011, 52, 6569; Ritter, O. M. S.; Giacomelli, F. C.; Passo, J. A.; Silveira, N. P.; Merlo, A. A.; Polym. Bull. 2006, $56,549$.

10. Tavares, A.; Livotto, P. R.; Gonçalves, P. F. B.; Merlo, A. A.; J. Braz. Chem. Soc. 2009, 20, 1742.

11. Finkelmann, H.; Ringsdorf, H.; Wendorff, J. H.; Makromol. Chem. 1978, 179, 273.

12. Palffy-Muhoray, P.; Nature 1998, 391, 745; Hu, J.-S.; Liu, C.; Zhang, X.; Meng, Q-B.; Eur. Polym. J. 2009, 45, 3292.

13. Merlo, A. A.; Ritter, O. M. S.; Pereira, F. V.; Vieira, C. H.; J. Braz. Chem. Soc. 2001, 12, 184; Merlo, A. A.; Gallardo, H.; Taylor, T. R.; Quim. Nova 2001, 24, 354.
14. Ely, F.; Hamanaka, M. H. O.; Mammana, A. P.; Quim. Nova 2007, 30, 1776; Shibaev, V. P.; Deussen, H. J.; Bobrovsky, A. Y.; Shibaev, P. V.; Boiko, N. I.; Borornholm, T.; Schaumburg, K.; Bechgaard, K.; Polym. Eng. Sci. 1997, 37, 6945; Craig, A. A.; Imrie, C. T.; J. Polym. Sci., Part A: Polym. Chem. 1996, 34, 421; Cowie, J. M. G.; Hunter, H. W.; Can. J. Chem. 1995, 73, 1811.

15. Donaldson, T.; Staesche, H.; Lu, Z. B.; Henderson, P. A.; Achard, M. F.; Imrie, C. T.; Liq. Cryst. 2010, 37, 1097.

16. Tavares, A.; Schneider, P. H.; Merlo, A. A.; Eur. J. Org. Chem. 2009, 2009, 889; Gordon, M. S.; Sojka, S. A.; Krause, J. G.; J. Org. Chem. 1984, 49, 2326; Owston, N. A.; Parker, A. J.; Williams, J. M. J.; Org. Lett. 2007, 9, 3599.

17. Passo, J. A.; Vilela, G. D.; Schneider, P. H.; Ritter, O. M. S.; Merlo, A. A.; Liq. Cryst. 2008, 35, 833.

18. Tavares, A.; Ritter, O. M. S.; Vasconcelos, U. B.; Arruda, B. C.; Schrader, A.; Schneider, P. H.; Merlo, A. A.; Liq. Cryst. 2010, 37, 159; Kamal, A.; Bharathi, E. V.; Reddy, J. S.; Ramaiah; M. J.; Dastagiri, D.; Reddy, M. K.; Viswanath, A.; Reddy, T. L.; Shaik, T. B.; Pushpavalli, S. N. C. V. L.; Bhadra, M. P.; Eur. J. Med. Chem. 2011, 46, 691; Hansen, E. C.; Levent, M.; Connolly, T. J.; Org. Process Res. Dev. 2010, 14, 574.

19. Colling, P. J.; Hird, M.; Introduction to Liquid Crystals. Chemistry and Physics, Taylor e Francis: Londres, 1997.

20. Silong, S.; Lutfor, M. R.; Rahman, M. Z. A.; Yunus, W. M. Z. W.; Haron, M. J.; Ahmad, M. B.; Yusoff, W. M. D. W.; J. Appl. Polym. Sci. 2002, 86, 2653; Imrie, C. T.; Attard, G. S.; Karasz, F. E.; Macromolecules 1996, 29, 1031.

21. Xu, X. Y.; Zhang, B. Y.; Wang, L. X.; Gu, W. M.; Liq. Cryst. 2009, 36, 1365; Hu, J.; Wei, K.; Zhang, B.; Yang, L.; Liq. Cryst. 2008, 35, 925; Severing, K.; Stibal-Fischer, E.; Hasenhindl, A.; Finkelmann, H.; Saalwächter, K.; J. Phys. Chem. B 2006, 110, 15680; Komiyat, Z.; Schrock , R. R.; Macromolecules 1993, 26, 1387; Chen, J.; Wang, X.; Zhang, H.; Wang, X.; Polym. Bull. 2005, 53, 223; Xie, M.; Zhang, C.; Liq. Cryst. 2007, 34, 1275 .

22. Hird, M.; Goodby, J. W.; Gough, N.; Toyne, K. J.; J. Mater. Chem., 2001, 11, 2732; Kang, S. K.; Samulski, E. T.; Kang, P.; Choo, J.; Liq Cryst. 2000, 27, 377.

23. Portugall, M.; Ringsdorf, H.; Zentel, R.; Makromol. Chem. 1982, 183, 2311; Craig, A. A.; Imrie, T. C.; Macromolecules 1999, 32, 6215.

24. Lee-Ruff, E.; Xi, F.; Qie, J. H.; J. Org. Chem. 1996, 61, 1547; HedinDahlström, J.; Shoravi, S.; Wikman, S.; Nicholls, I. A.; Tetrahedron: Asymmetyry 2004, 15, 2431; Bobrovsky, A. Y.; Boiko, N. I.; Shibaev, V. P.; Wolff, D.; Springer, J.; Macromolecules 1998, 31, 5800; Hu, J.; Zhang, B.; Pan, W.; Zhou, A.; Liq. Cryst. 2005, 32, 441. 


\section{SÍNTESE E CARACTERIZAÇÃO DE COPOLÍMEROS DE CADEIA LATERAL DERIVADOS DE ACRILATOS DE} 4,5-DI-HIDROISOXAZOL E DO (-)-MENTOL

Joel A. Passo e Aloir A. Merlo*\#

Instituto de Química, Universidade Federal do Rio Grande do Sul, Av. Bento Gonçalves, 9500, 91501-970 Porto Alegre - RS, Brasil Juliana Eccher e Ivan H. Bechtold

Departamento de Física, Universidade Federal de Santa Catarina, Campus Universitário Trindade, 88040-900 Florianópolis - SC, Brasil

\section{Stephen M. Kelly}

Department of Chemistry, University of Hull, U67RX, Hull, UK

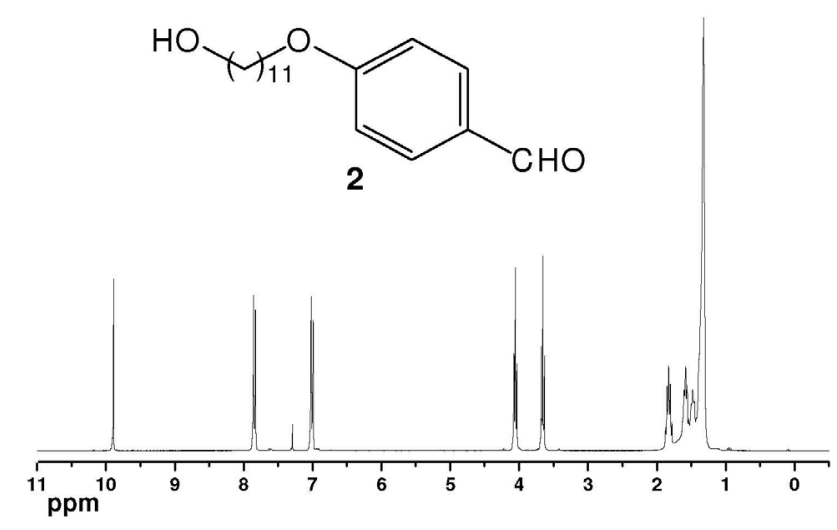

Figura 1S. Espectro de $\mathrm{RMN}^{1} \mathrm{H}\left(\mathrm{CDCl}_{3}\right), 300 \mathrm{MHz}$ do aldeído 2

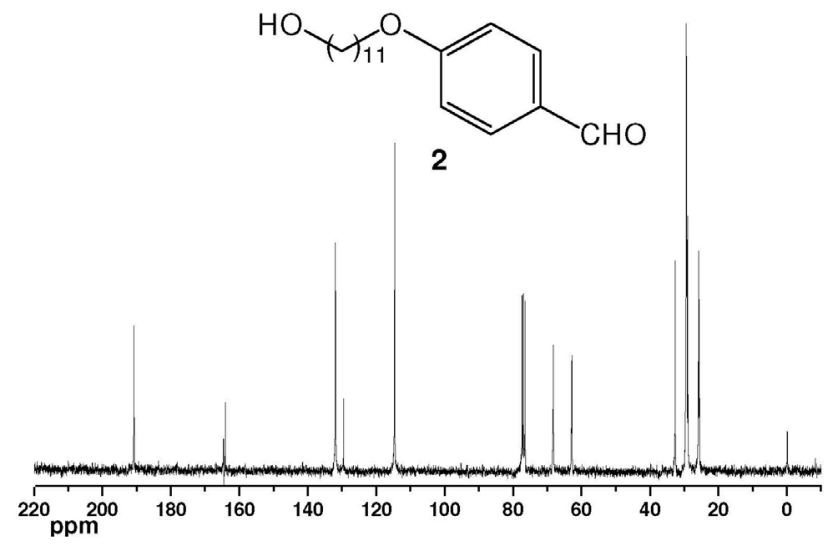

Figura 2S. Espectro de $\mathrm{RMN}^{13} \mathrm{C}\left(\mathrm{CDCl}_{3}\right), 75 \mathrm{MHz}$ do aldeído 2

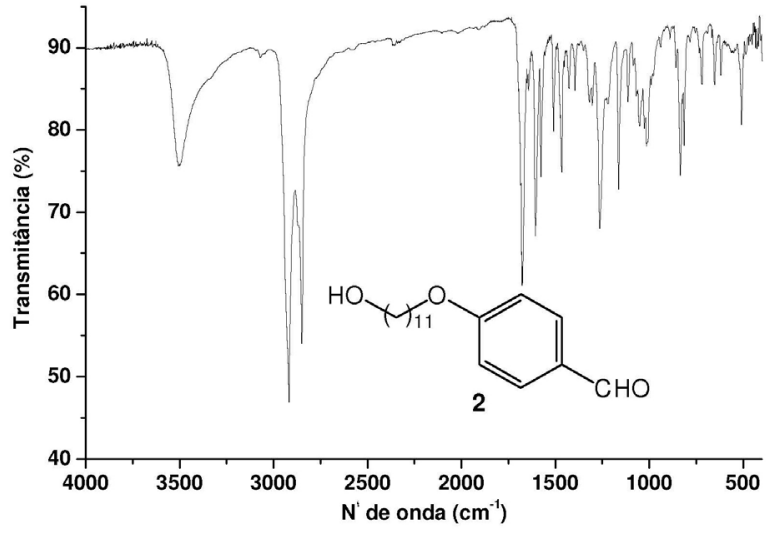

Figura 3S. Espectro de Infravermelho do aldeído 2
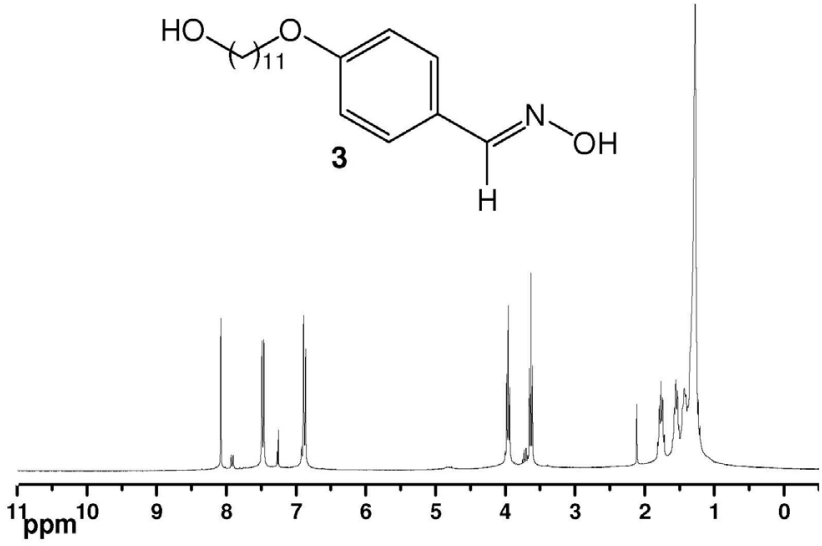

Figura 4S. Espectro de $\mathrm{RMN}^{1} \mathrm{H}\left(\mathrm{CDCl}_{3}\right), 300 \mathrm{MHz}$ da oxima 3 


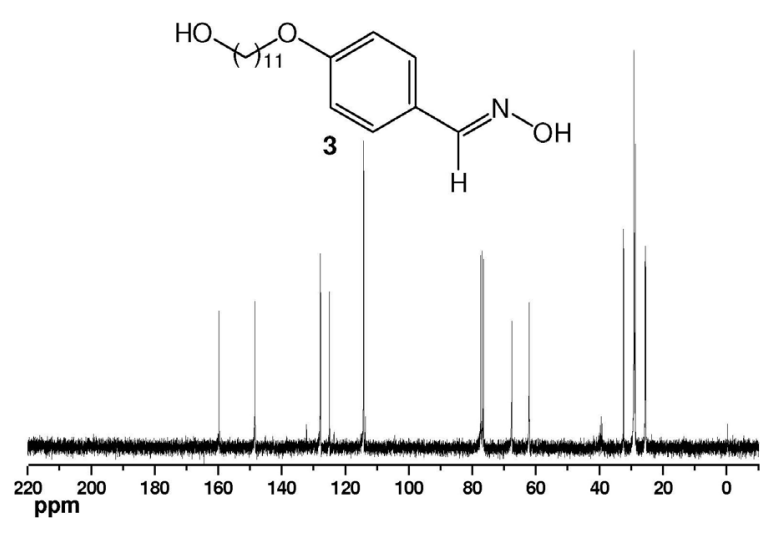

Figura 5S. Espectro de $\mathrm{RMN}^{13} \mathrm{C}\left(\mathrm{CDCl}_{3}\right), 75 \mathrm{MHz}$ da oxima 3

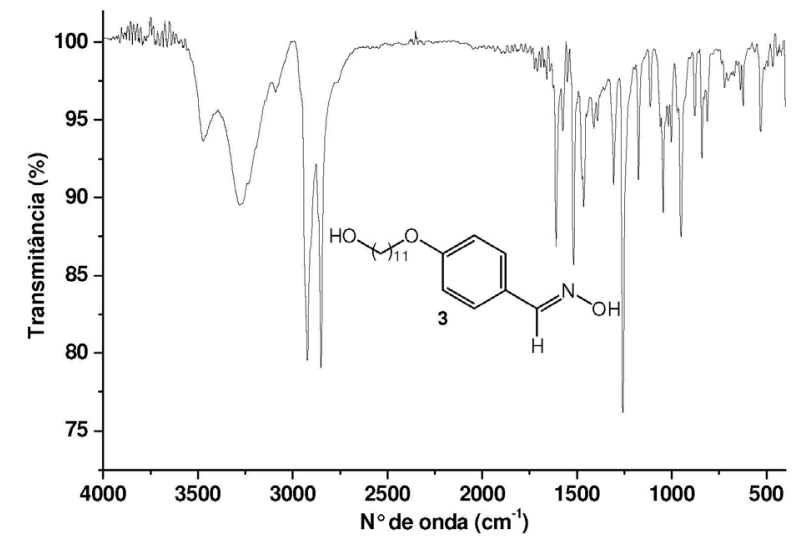

Figura 6S. Espectro de Infravermelho da oxima 3

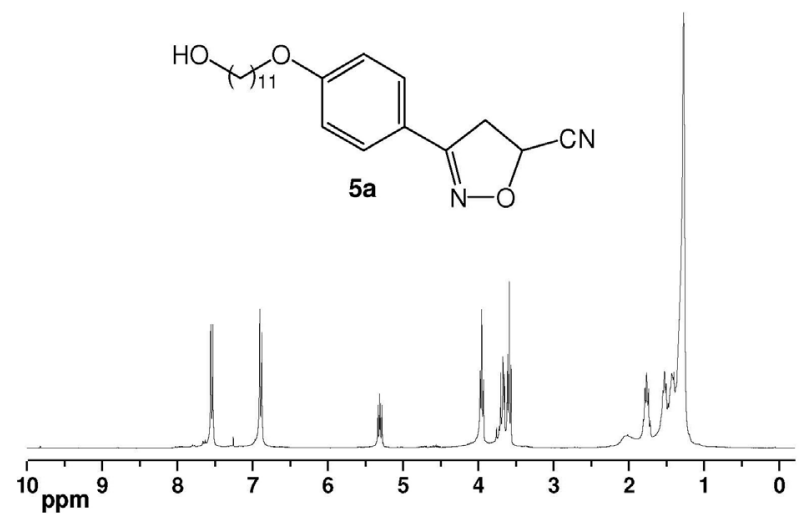

Figura 7S. Espectro de $\mathrm{RMN}^{1} \mathrm{H}\left(\mathrm{CDCl}_{3}\right), 300 \mathrm{MHz}$ da isoxazolina $\mathbf{5 a}$

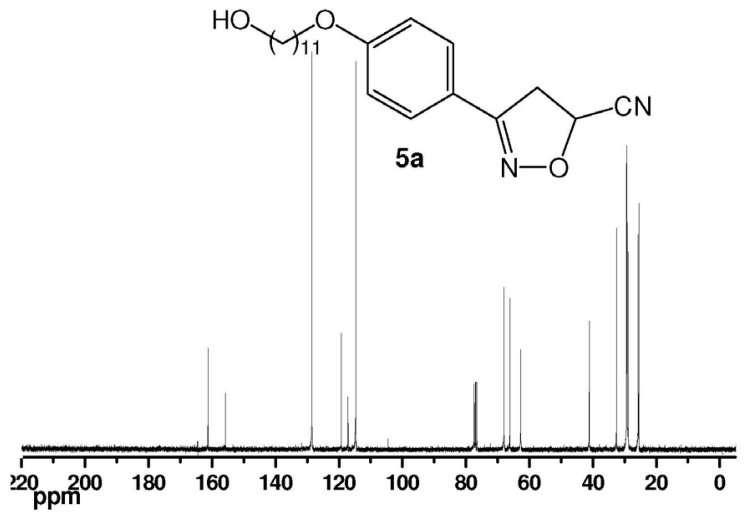

Figura 8S. Espectro de $\mathrm{RMN}^{13} \mathrm{C}\left(\mathrm{CDCl}_{3}\right), 75 \mathrm{MHz}$ da isoxazolina 5 a

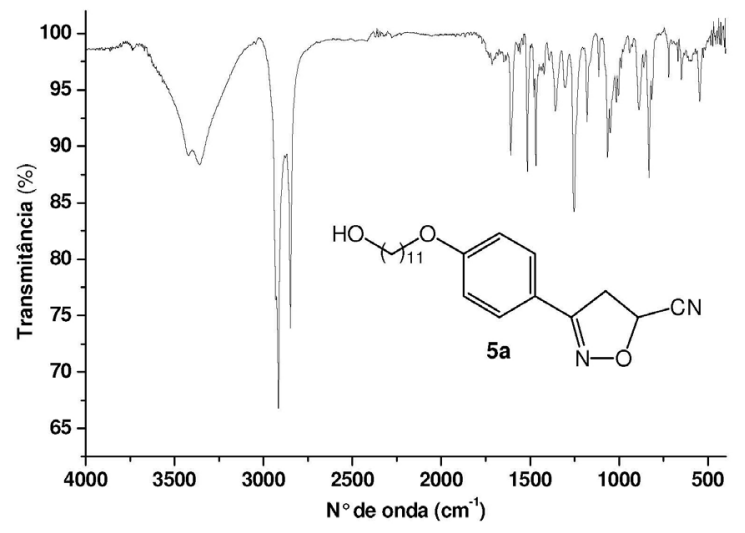

Figura 9S. Espectro de Infravermelho da isoxazolina 5 a

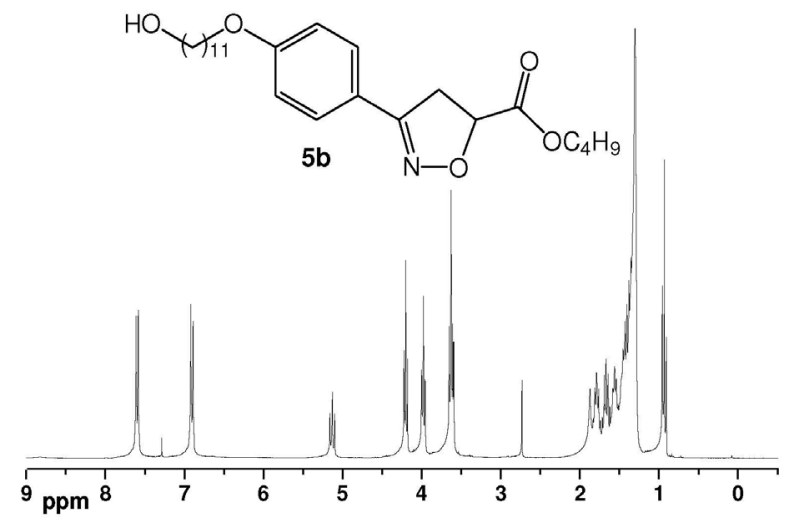

Figura 10S. Espectro de $\mathrm{RMN}^{l} \mathrm{H}\left(\mathrm{CDCl} \mathrm{Cl}_{3} / \mathrm{DMSO}_{d 6}\right), 300 \mathrm{MHz}$ da isoxazolina $5 b$ 


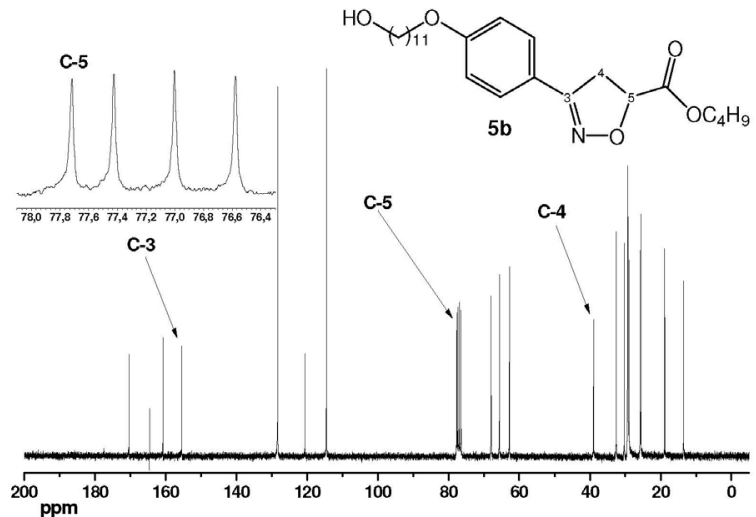

Figura 11S. Espectro de $\mathrm{RMN}^{13} \mathrm{C}\left(\mathrm{CDCl}_{3} / \mathrm{DMSO} \mathrm{O}_{d 6}\right), 75 \mathrm{MHz}$ da isoxazolina $5 \boldsymbol{b}$

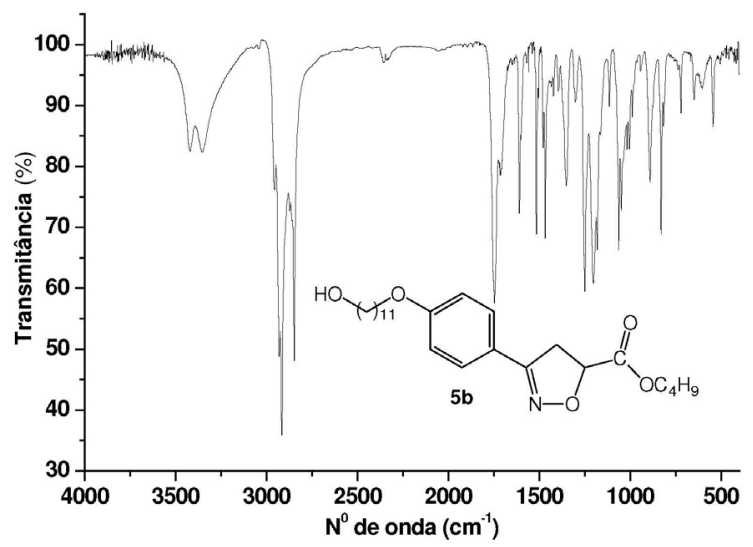

Figura 12S. Espectro de Infravermelho da isoxazolina $\mathbf{5 b}$

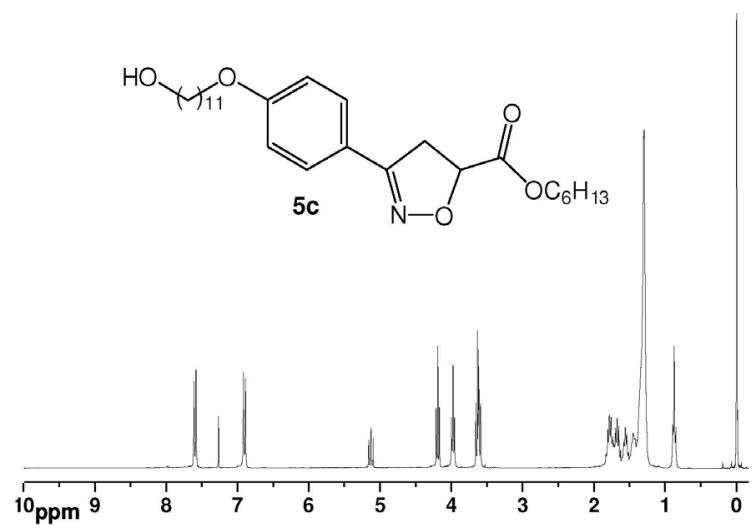

Figura 13S. Espectro de $\mathrm{RMN}^{1} \mathrm{H}\left(\mathrm{CDCl}_{3}\right), 300 \mathrm{MHz}$ da isoxazolina $5 \mathrm{c}$<smiles>CCCCCOC(=O)C1CC(c2ccc(O[14CH3])cc2)=NO1</smiles>

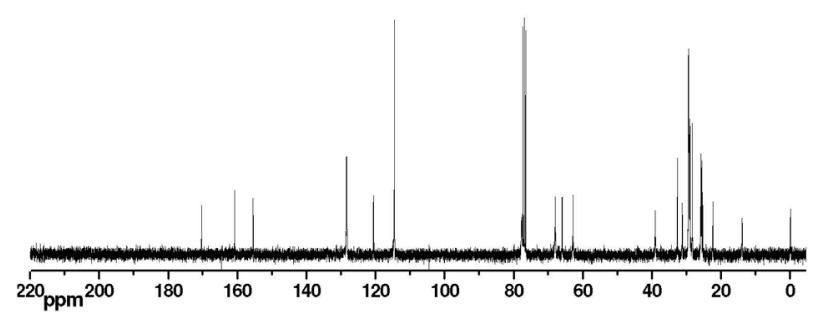

Figura 14S. Espectro de $\mathrm{RMN}^{13} \mathrm{C}\left(\mathrm{CDCl}_{3}\right), 75 \mathrm{MHz}$ da isoxazolina $5 \mathrm{c}$

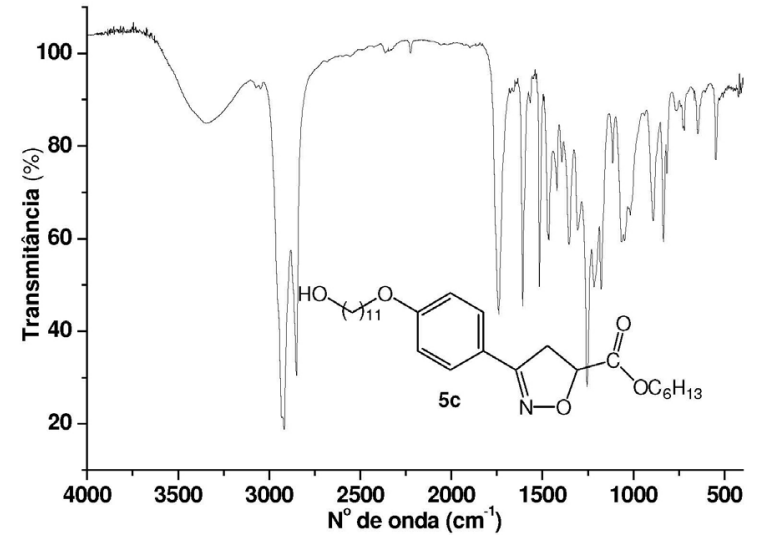

Figura 15S. Espectro de Infravermelho da isoxazolina $5 c$<smiles>O=C(O)C1CC(c2ccc(OCO)cc2)=NO1</smiles>

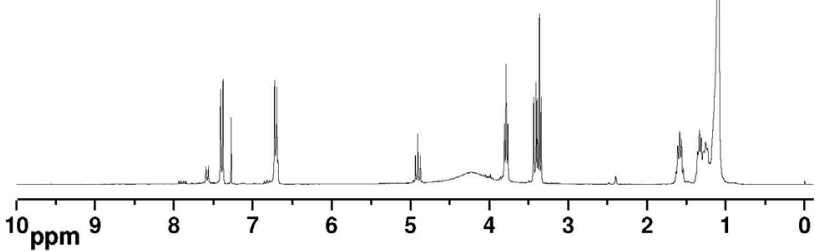

Figura 16S. Espectro de $\mathrm{RMN}^{1} \mathrm{H}\left(\mathrm{CDCl}_{3} / \mathrm{DMSO}_{d 6}\right), 300 \mathrm{MHz}$ da isoxazolina $5 d$ 


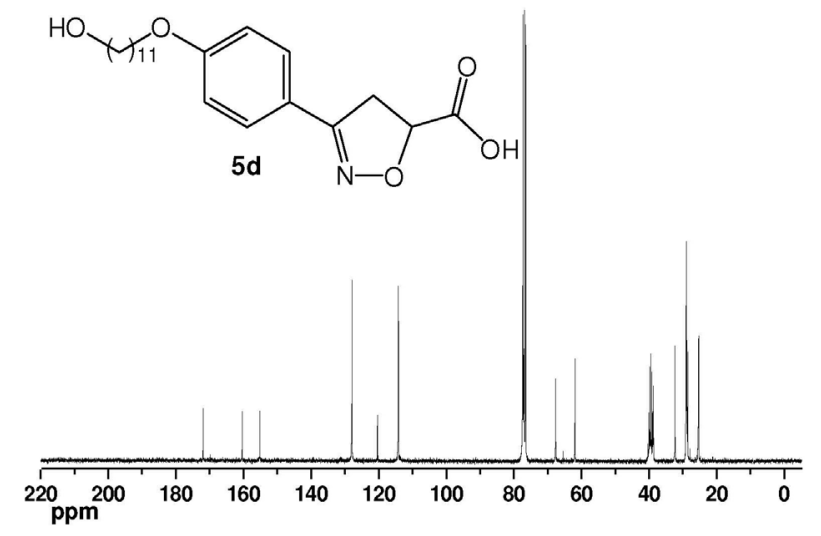

Figura 17S. Espectro de $\mathrm{RMN}^{13} \mathrm{C}\left(\mathrm{CDCl}_{3} / \mathrm{DMSO}_{d 6}\right), 75 \mathrm{MHz}$ da isoxazolina $5 d$

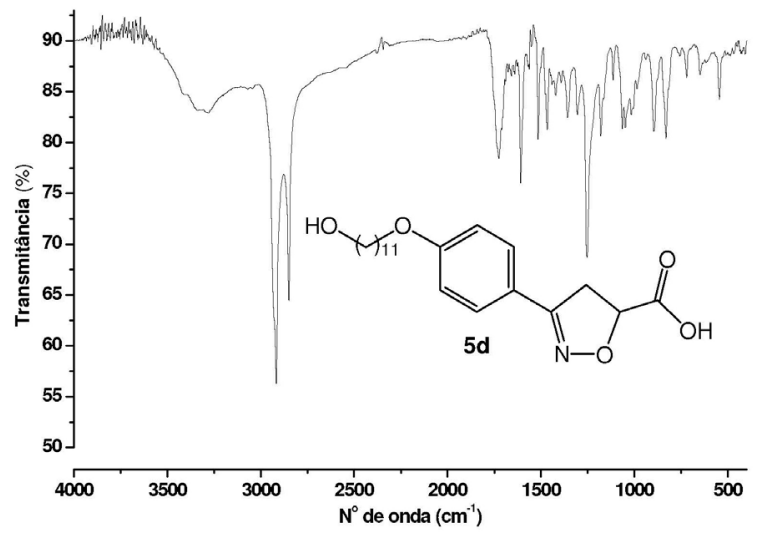

Figura 18S. Espectro de Infravermelho da isoxazolina 5 d

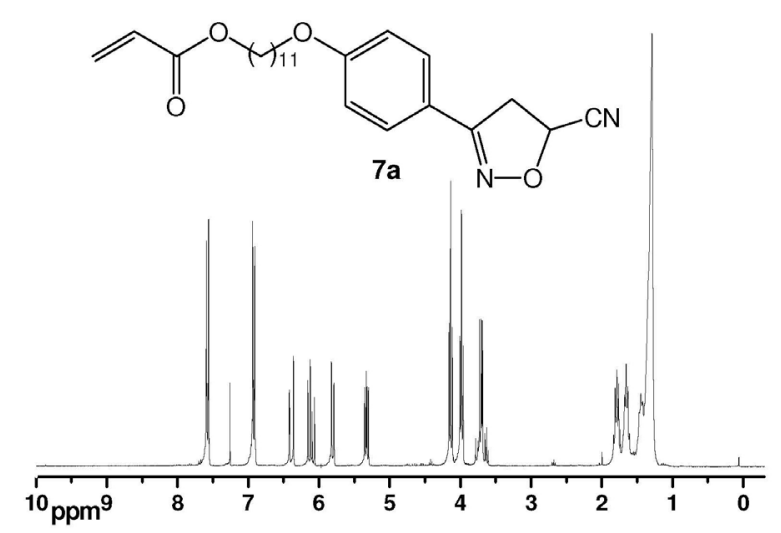

Figura 19S. Espectro de $\mathrm{RMN}^{1} \mathrm{H}\left(\mathrm{CDCl}_{3} / \mathrm{DMSO}_{d 6}\right), 300 \mathrm{MHz}$ do monômero $7 \mathrm{a}$

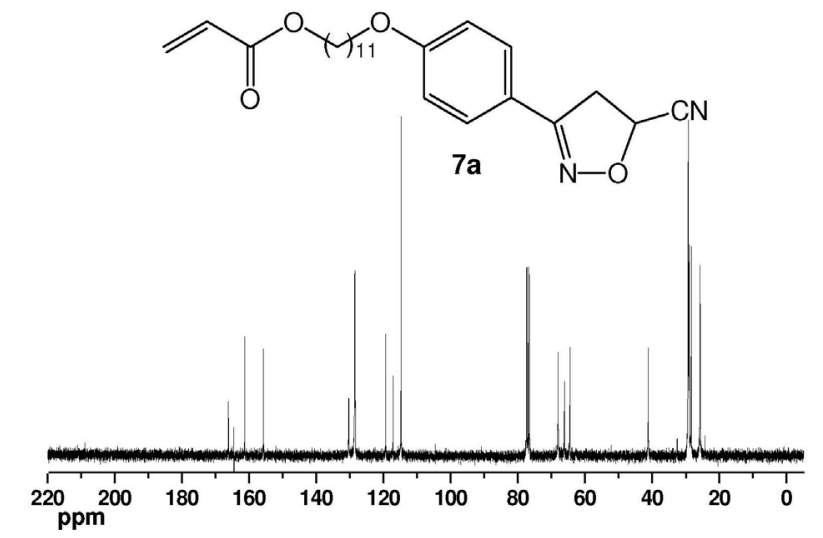

Figura 20S. Espectro de $\mathrm{RMN}^{13} \mathrm{C}\left(\mathrm{CDCl}_{3} / \mathrm{DMSO}_{d 6}\right), 75 \mathrm{MHz}$ do monômero 7 a

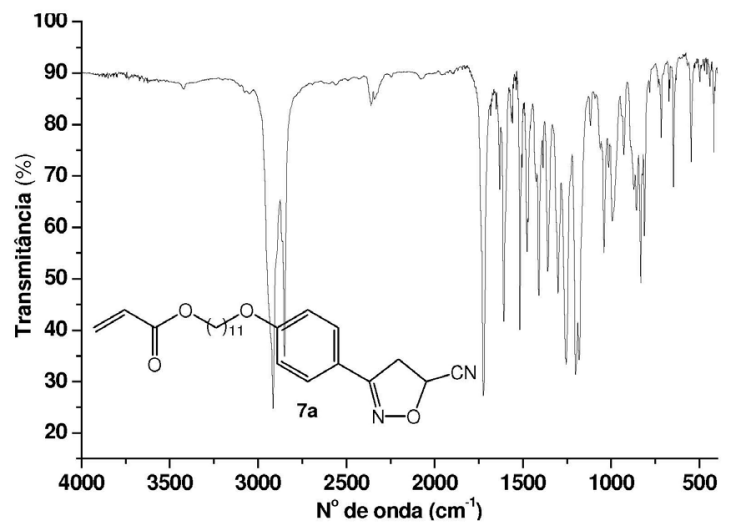

Figura 21S. Espectro de Infravermelho do monômero 7 a

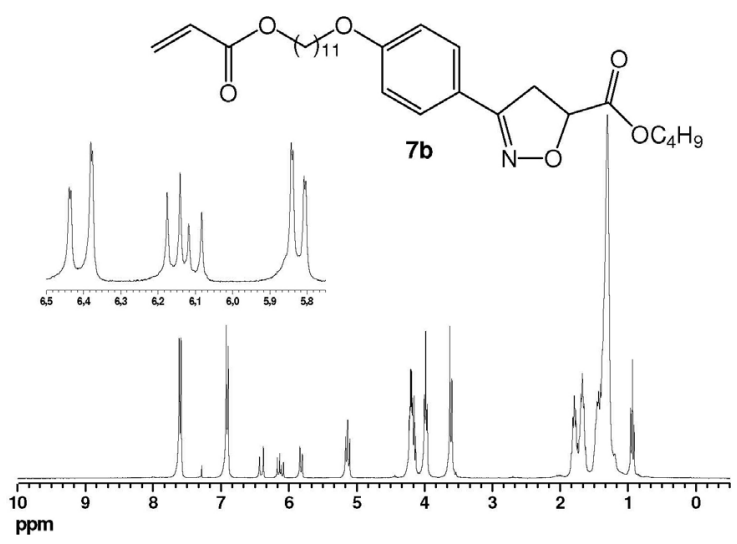

Figura 22S. Espectro de $\mathrm{RMN}^{1} \mathrm{H}\left(\mathrm{CDCl}_{3}\right), 300 \mathrm{MHz}$ do monômero $7 \mathrm{~b}$ 


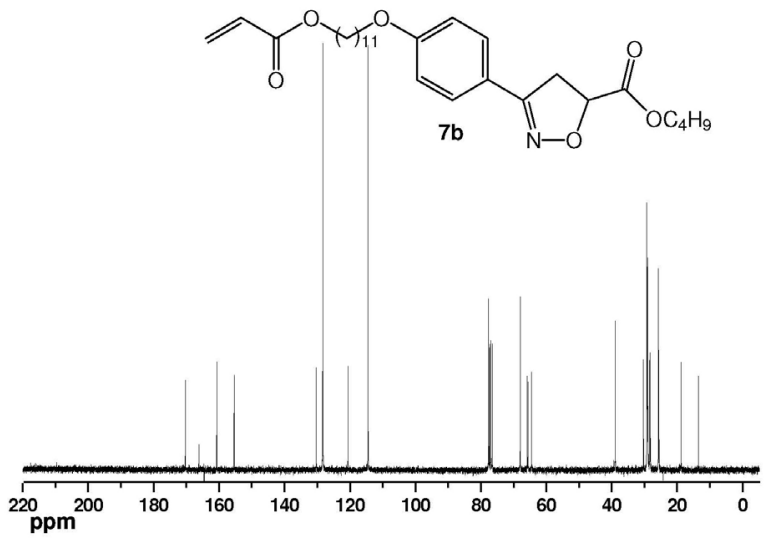

Figura 23S. Espectro de $\mathrm{RMN}^{13} \mathrm{C}\left(\mathrm{CDCl}_{3}\right), 75 \mathrm{MHz}$ do monômero $7 \boldsymbol{b}$

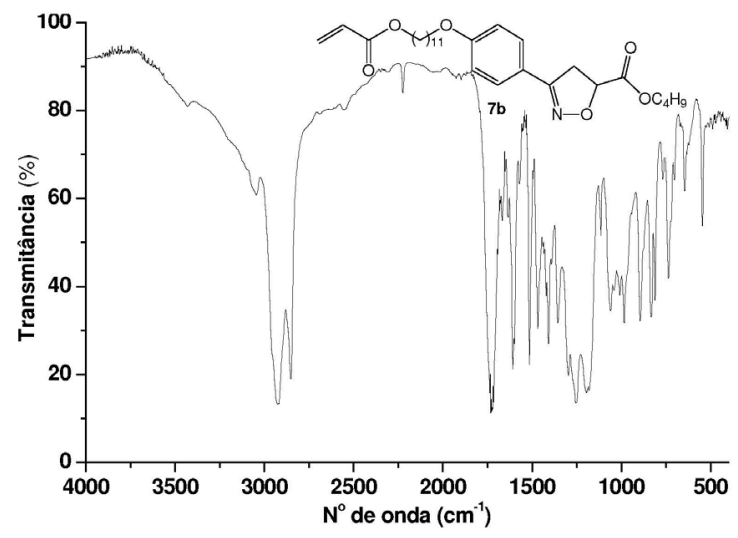

Figura 24S. Espectro de Infravermelho do monômero $7 \boldsymbol{b}$

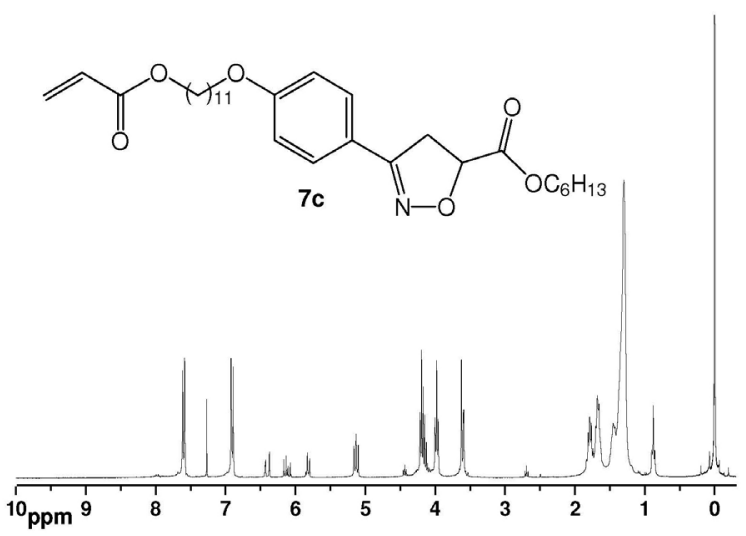

Figura 25S. Espectro de $\mathrm{RMN}^{l} \mathrm{H}\left(\mathrm{CDCl}_{3} / \mathrm{DMSO}_{d 6}\right), 300 \mathrm{MHz}$ do monômero $7 \mathrm{c}$

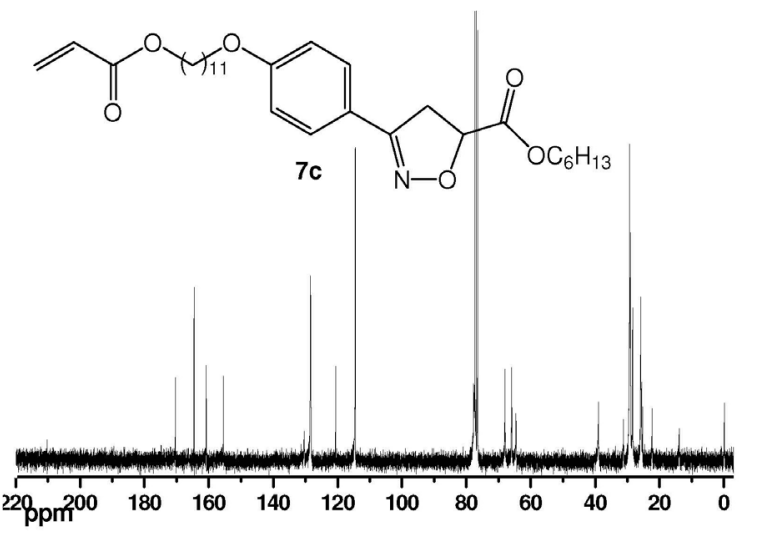

Figura 26S. Espectro de $\mathrm{RMN}^{13} \mathrm{C}\left(\mathrm{CDCl}_{3} / \mathrm{DMSO}_{d 6}\right), 75 \mathrm{MHz}$ do monômero $7 \mathrm{c}$

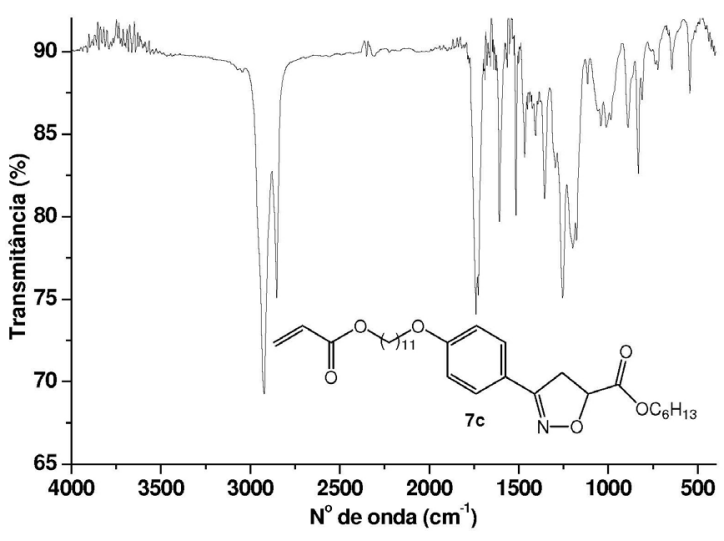

Figura 27S. Espectro de Infravermelho do monômero 7 c

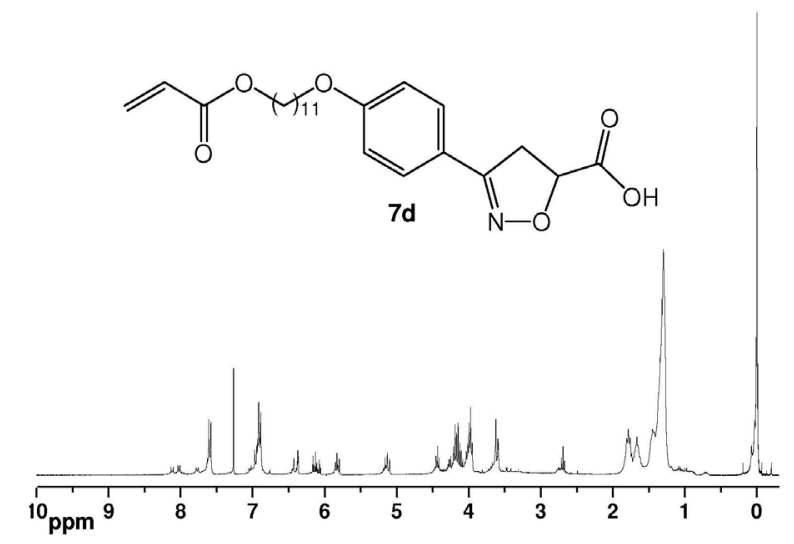

Figura 28S. Espectro de $\mathrm{RMN}^{l} \mathrm{H}\left(\mathrm{CDCl}_{3} \mathrm{DMSO} \mathrm{d}_{d 6}\right), 300 \mathrm{MHz}$ do monômero $7 d$ 


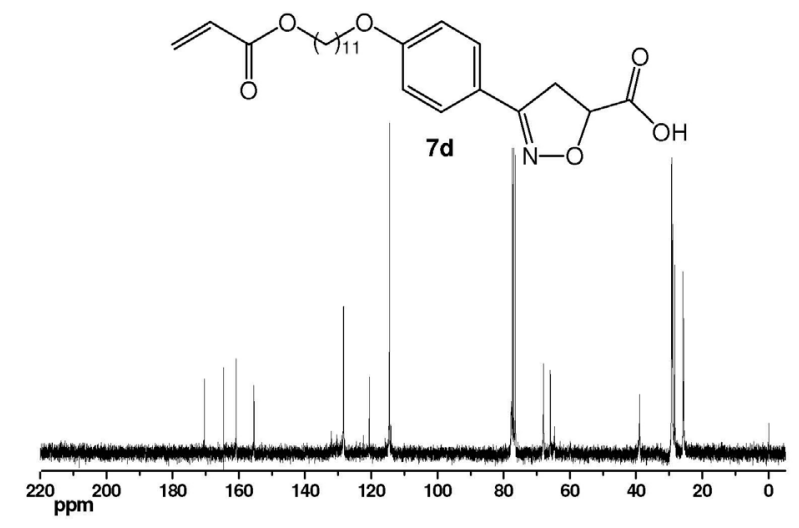

Figura 29S. Espectro de $\mathrm{RMN}^{13} \mathrm{C}\left(\mathrm{CDCl}_{3} / \mathrm{DMSO}_{d 6}\right), 75 \mathrm{MHz}$ do monômero $7 d$

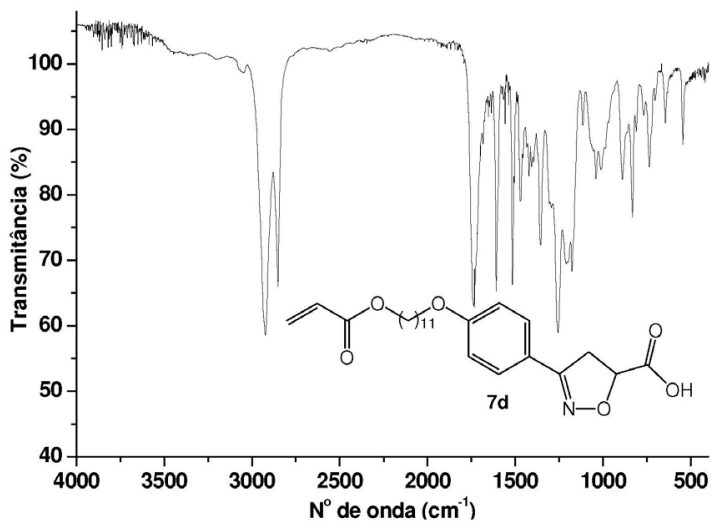

Figura 30S. Espectro de Infravermelho do monômero 7d<smiles>C=CC(=O)O[C@H]1C[C@H](C)CC[C@H]1C(C)C</smiles>

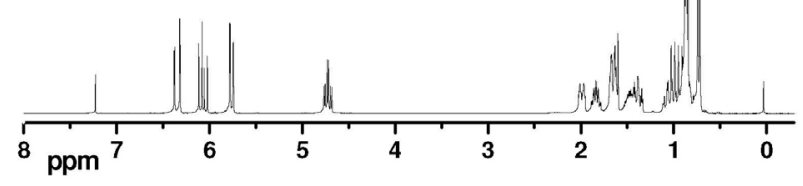

Figura 31S. Espectro de $\mathrm{RMN}^{1} \mathrm{H}\left(\mathrm{CDCl}_{3}\right), 300 \mathrm{MHz}$ do monômero 9

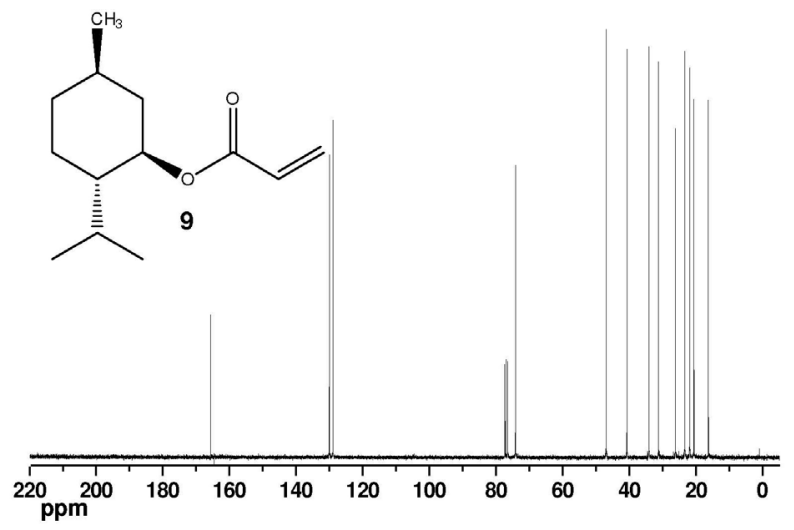

Figura 32S. Espectro de $\mathrm{RMN}^{13} \mathrm{C}\left(\mathrm{CDCl}_{3}\right), 75 \mathrm{MHz}$ do monômero 9

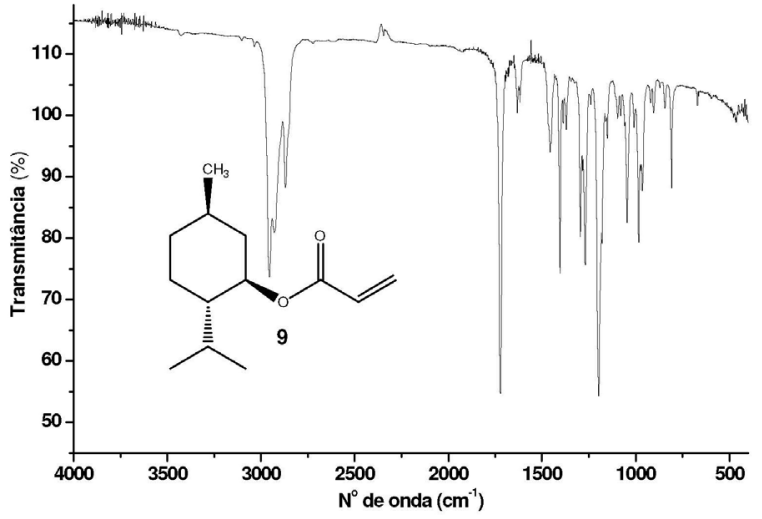

Figura 33S. Espectro de Infravermelho do monômero 9
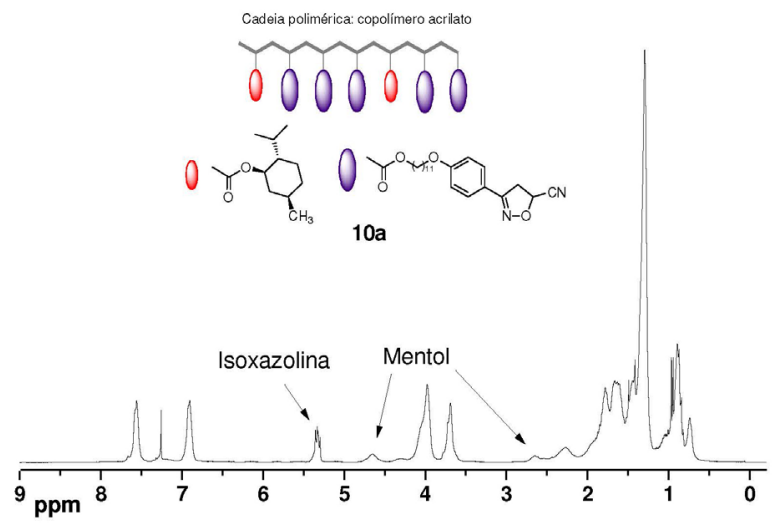

Figura 34S. Espectro de $\mathrm{RMN}^{l} \mathrm{H}\left(\mathrm{CDCl}_{3}\right), 300 \mathrm{MHz}$ do copolímero $10 \mathrm{a}$ 


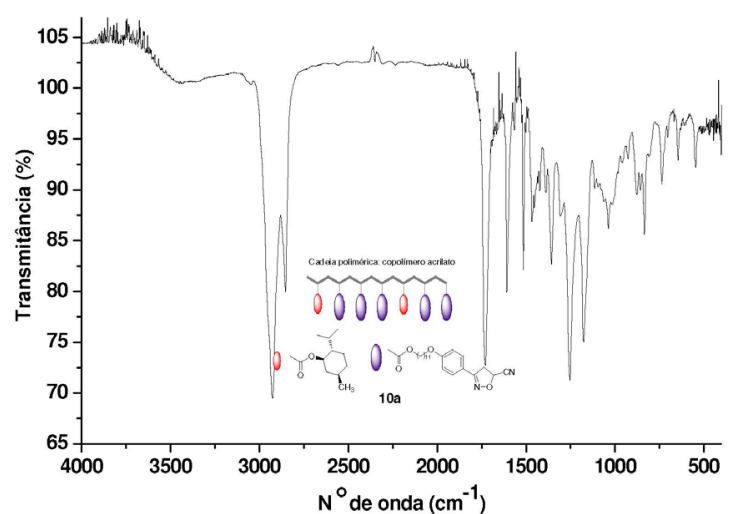

Figura 35S. Espectro de Infravermelho do copolímero 10 a

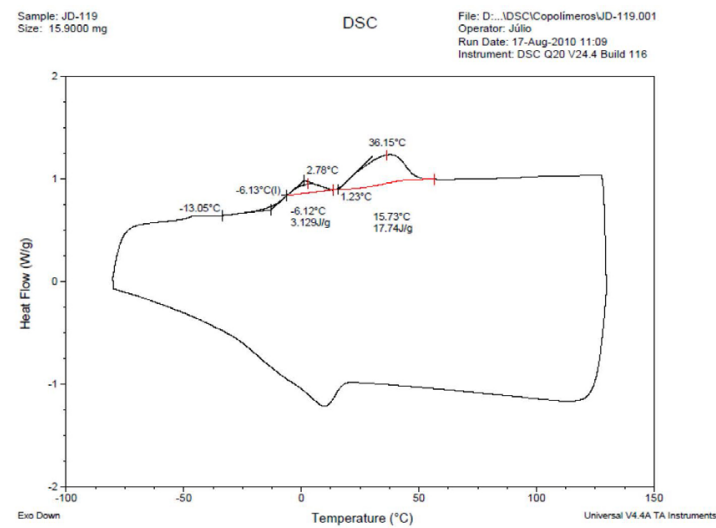

Figura 36S. Termograma de DSC do copolímero $10 a-2^{\circ}$ ciclo $\left(20^{\circ} \mathrm{C} / \mathrm{min}\right)$

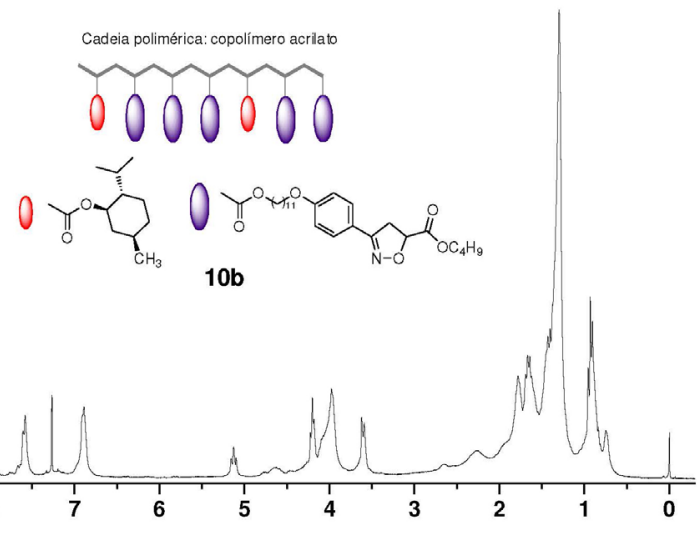

Figura 37S. Espectro de $\mathrm{RMN}^{\mathrm{I}} \mathrm{H}\left(\mathrm{CDCl}_{3}\right), 300 \mathrm{MHz}$ do copolímero $10 \mathrm{~b}$

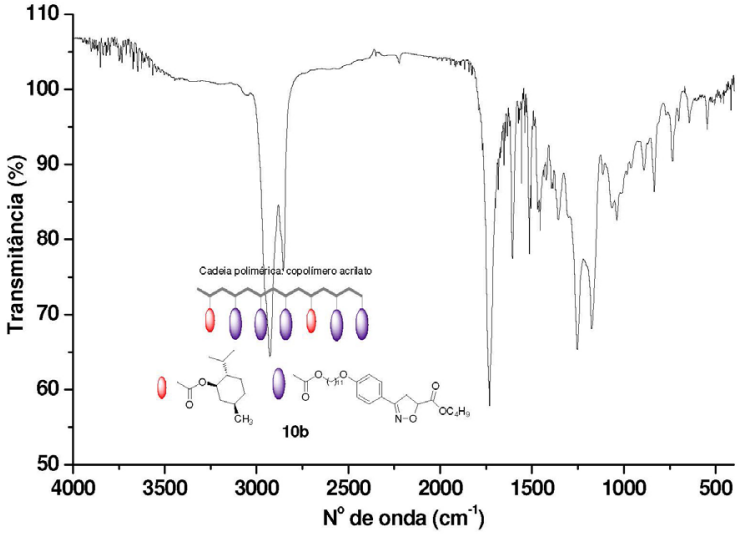

Figura 38S. Espectro de Infravermelho do copolímero $10 \mathrm{~b}$

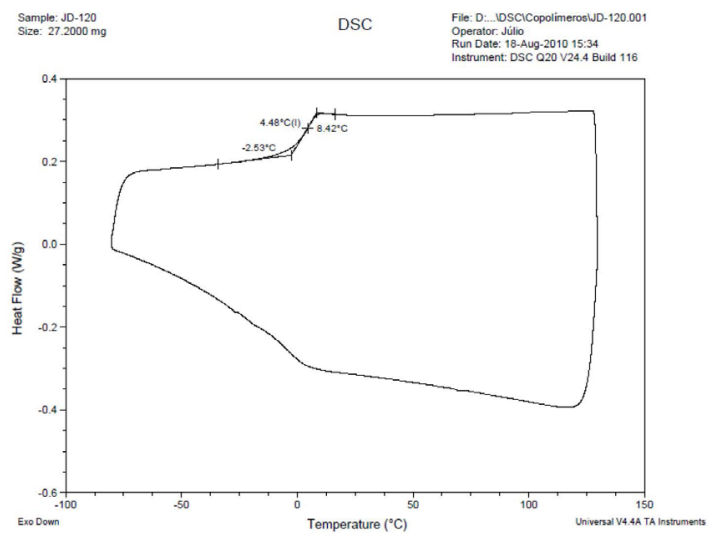

Figura 39S. Termograma de DSC do copolímero $10 \mathrm{~b}-2^{\circ}$ ciclo $\left(20^{\circ} \mathrm{C} / \mathrm{min}\right)$

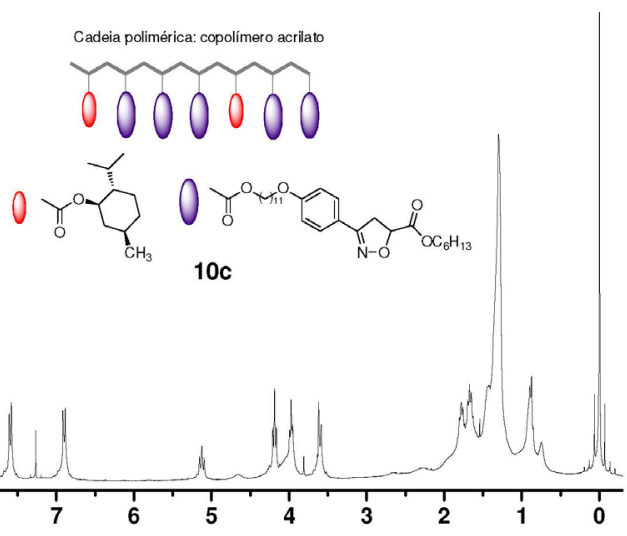

Figura 40S. Espectro de $\mathrm{RMN}^{1} \mathrm{H}\left(\mathrm{CDCl}_{3}\right), 300 \mathrm{MHz}$ do copolímero $10 \mathrm{c}$ 


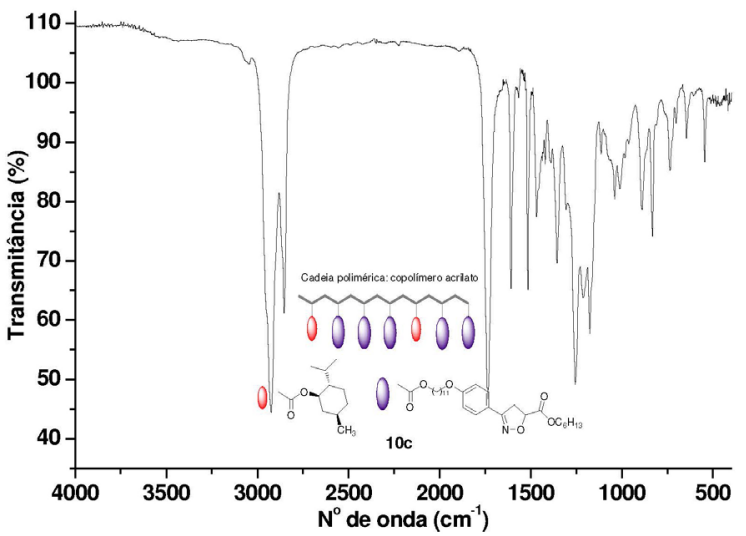

Figura 41S. Espectro de Infravermelho do copolímero $10 \mathrm{c}$

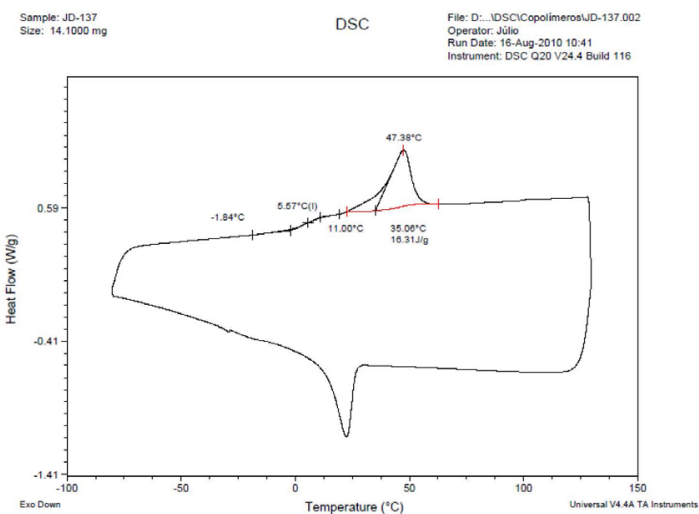

Figura 42S. Termograma de DSC do copolímero $10 \mathrm{c}-2^{\circ}$ ciclo $\left(20^{\circ} \mathrm{C} / \mathrm{min}\right)$

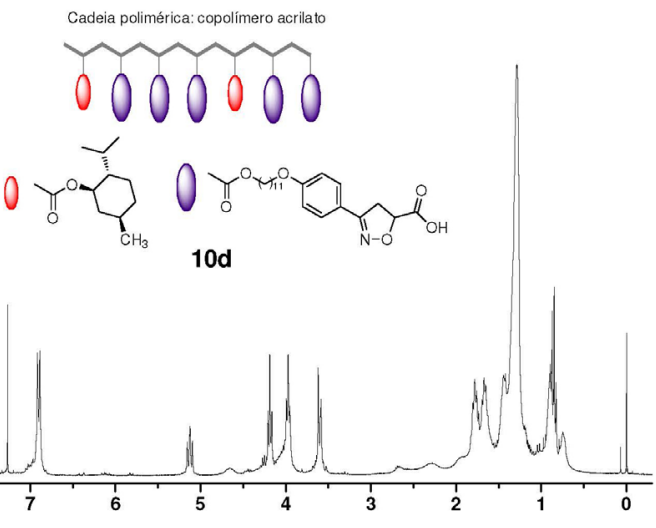

Figura 43S. Espectro de $\mathrm{RMN}^{1} \mathrm{H}\left(\mathrm{CDCl}_{3}\right), 300 \mathrm{MHz}$ do copolímero $10 \mathrm{~d}$

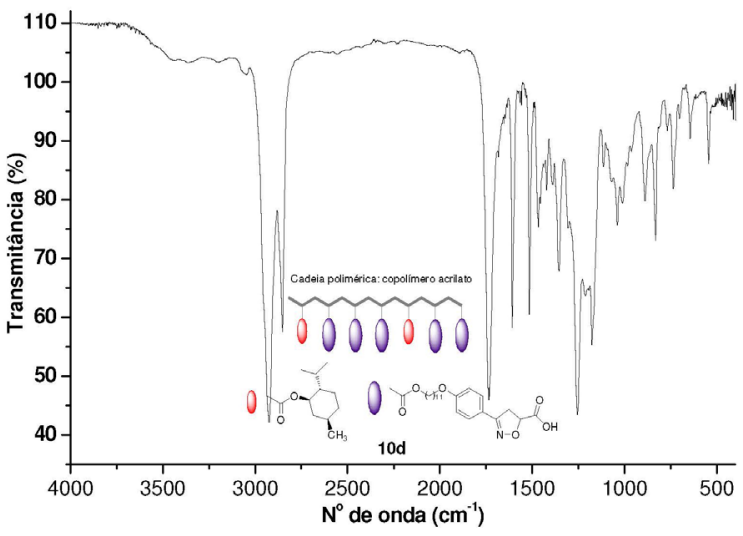

Figura 44S. Espectro de Infravermelho do copolímero 10d

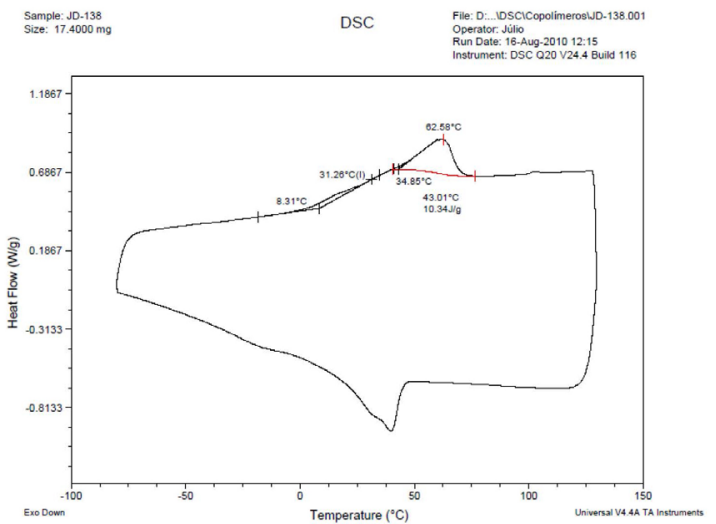

Figura 45S. Termograma de DSC do copolímero $10 d-2^{\circ}$ ciclo $\left(20^{\circ} \mathrm{C} / \mathrm{min}\right)$

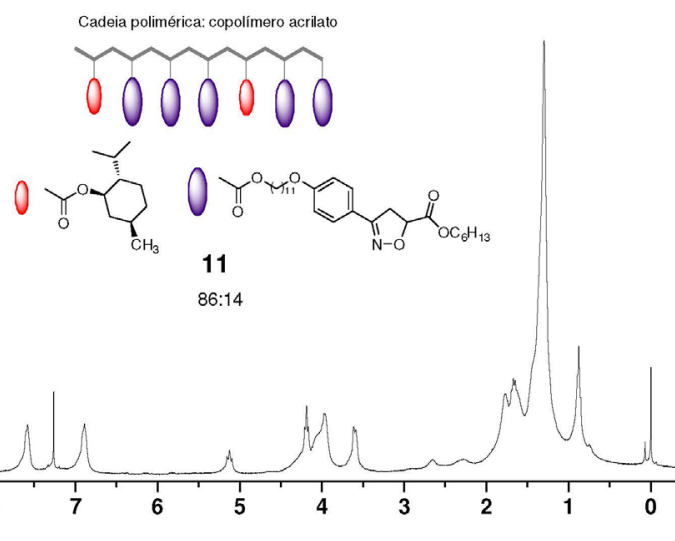

Figura 46S. Espectro de $\mathrm{RMN}^{1} \mathrm{H}\left(\mathrm{CDCl}_{3}\right), 300 \mathrm{MHz}$ do copolímero 11 


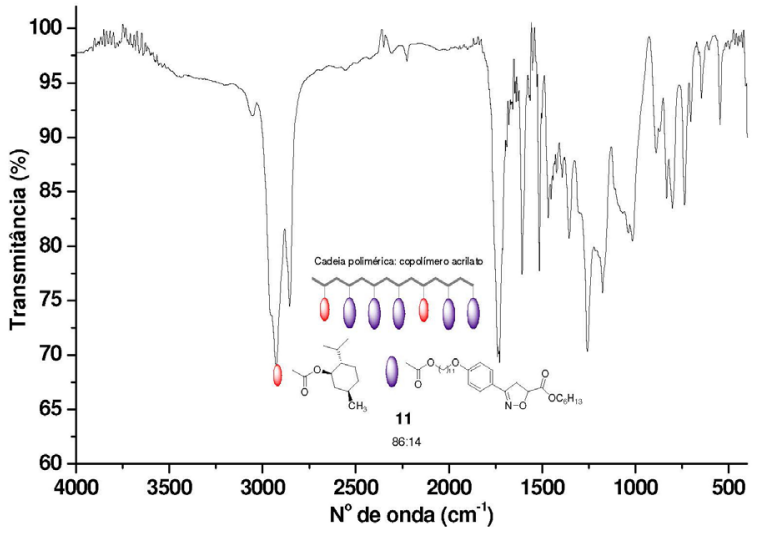

Figura 47S. Espectro de Infravermelho do copolímero 11

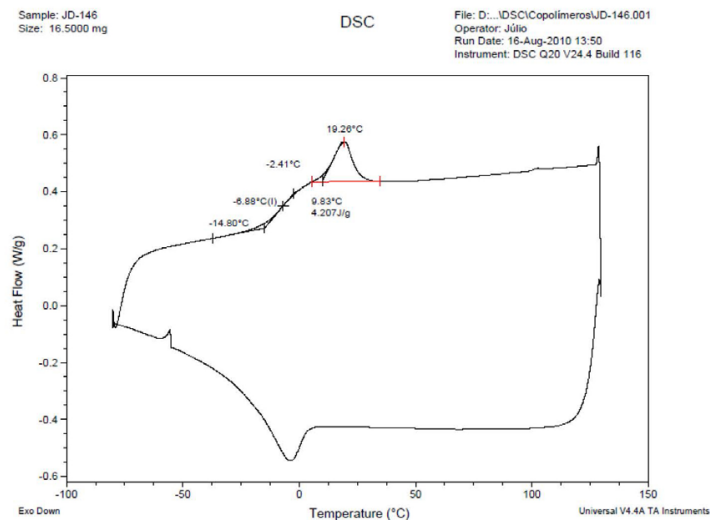

Figura 48S. Termograma de DSC do copolímero $11-2^{\circ}$ ciclo $\left(20^{\circ} \mathrm{C} / \mathrm{min}\right)$

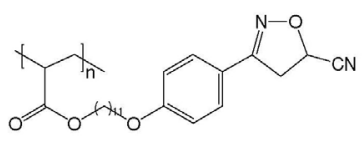

12

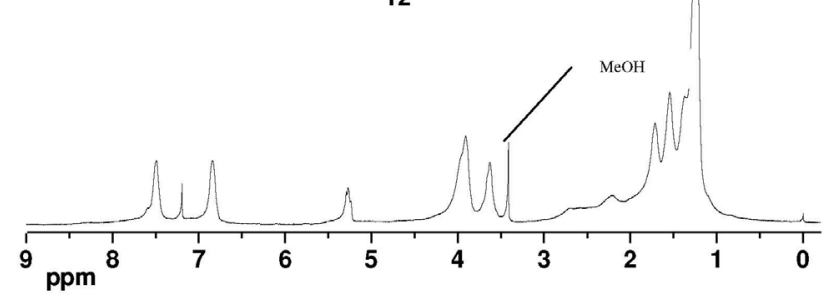

Figura 49S. Espectro de $\mathrm{RMN}^{1} \mathrm{H}\left(\mathrm{CDCl}_{3}\right), 300 \mathrm{MHz}$ do homopolímero 12

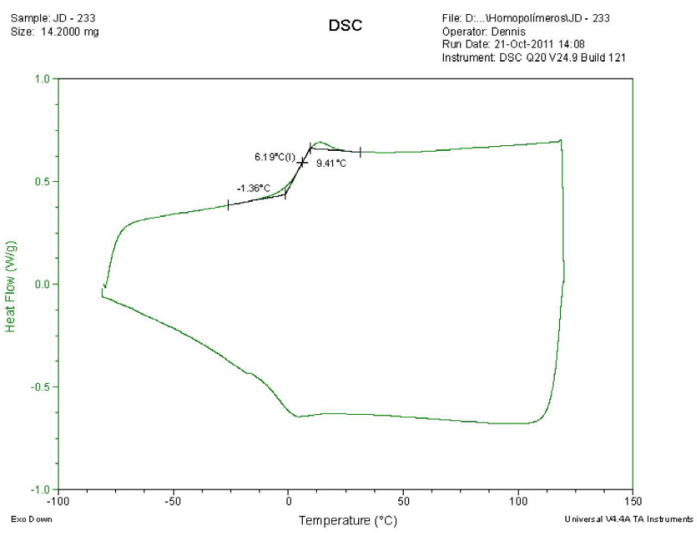

Figura 50S. Termograma de DSC do copolímero $12-2^{\circ}$ ciclo $\left(20^{\circ} \mathrm{C} / \mathrm{min}\right)$

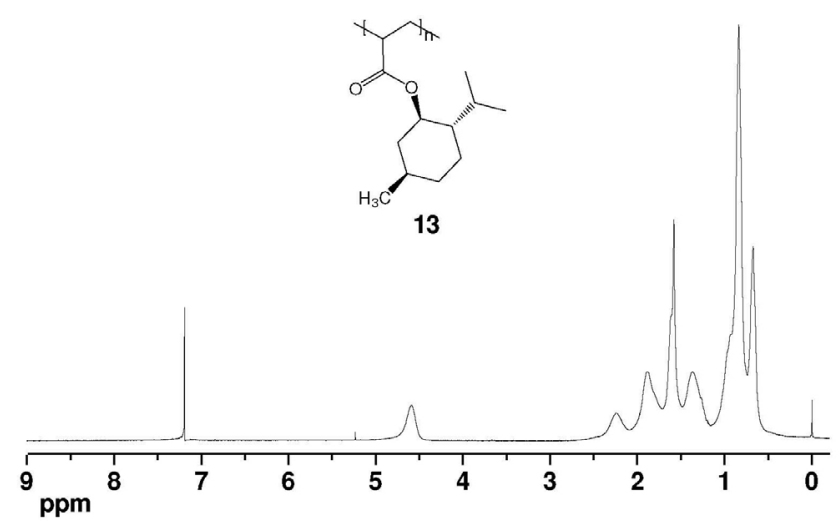

Figura 51S. Espectro de $\mathrm{RMN}^{l} \mathrm{H}\left(\mathrm{CDCl}_{3}\right), 300 \mathrm{MHz}$ do homopolímero 13

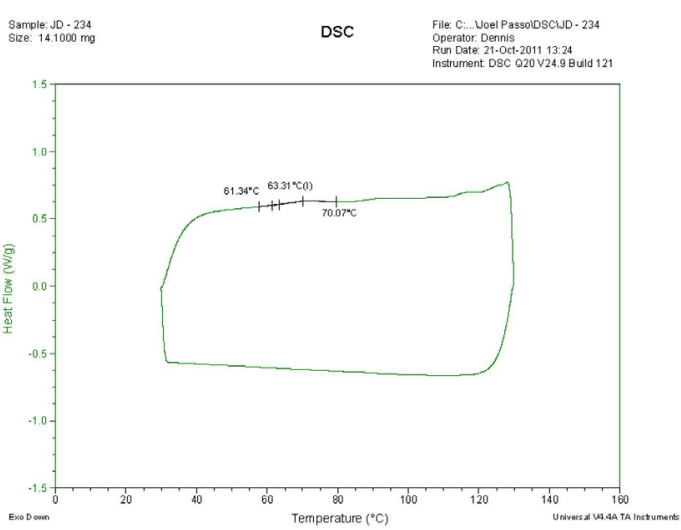

Figura 52S. Termograma de DSC do homolímero $13-1^{\circ}$ ciclo $\left(20^{\circ} \mathrm{C} / \mathrm{min}\right)$ 